\title{
A WEB-BASED LEARNING SUPPORT SYSTEM FOR ROUGH SETS
}

\author{
A Thesis \\ Submitted to the Faculty of Graduate Studies and Research \\ In Partial Fulfillment of the Requirements \\ FOR THE DEGREE OF \\ MASTER OF SCIENCE \\ IN \\ Computer Science \\ UNIVERSITY OF REGINA \\ By \\ Ying Zhou \\ Regina, Saskatchewan \\ August 2014
}

Copyright (c) 2014: Ying Zhou 


\section{UNIVERSITY OF REGINA}

\section{FACULTY OF GRADUATE STUDIES AND RESEARCH SUPERVISORY AND EXAMINING COMMITTEE}

Ying Zhou, candidate for the degree of Master of Science in Computer Science, has presented a thesis titled, $\boldsymbol{A}$ Web-Based Learning Support System for Rough Sets, in an oral examination held on August 18, 2014. The following committee members have found the thesis acceptable in form and content, and that the candidate demonstrated satisfactory knowledge of the subject material.

External Examiner: $\quad$ Dr. Yasser Morgan, Software Systems Engineering

Supervisor: $\quad$ Dr. Jingtao Yao, Department of Computer Science

Committee Member: Dr. Lawrence V. Saxton, Department of Computer Science

Committee Member: $\quad$ Dr. Lisa Fan, Department of Computer Science

Chair of Defense: $\quad$ Dr. Chun-Hua Guo, Department of Mathematics \& Statistics 


\section{Abstract}

Web-based learning is gaining popularity due to its convenience, ubiquity, personalization, and adaptation features compared with traditional learning environments. The learning subjects of Web-based learning systems are mostly for popular sciences. Little attention has been paid for learning cutting edge subjects and no such systems have been developed for rough sets.

Rough set theory has obtained significant attention as a mathematical approach to data representation and analysis in recent years. To realize Web-based learning for this subject, Web-based learning support systems can provide a reasonable framework. Web-based learning support systems sustain teaching as well as learning by making use of the Web technology, and are able to offer student-centered education.

This thesis presents the design principle, system architectures, and prototype implementation of a Web-based learning support system named Online Rough Sets (ORS). The system is specifically designed for learning rough sets in a studentcentered learning environment. Some special features, such as adaptation, are emphasized in the system. The ORS has the ability of adaptation to student preference and performance by modifying the size and order of learning materials delivered to each individual. Additionally, it predicts estimated learning time of each topic, which is helpful for students to schedule their learning paces. Two demonstrative examples show ORS can support students to learn rough sets rationally and efficiently. 


\section{Acknowledgments}

I would express deep gratitude to my supervisor Dr. JingTao Yao for his patience and guidance in carrying out this thesis. This thesis would have not been possible without his consistent support. It is a great pleasure and honour for me to conduct this research under his supervision.

In addition to my supervisor, I wish to express my appreciation to the supervisory committee, i.e., Dr. Larry Saxton and Dr. Lisa Fan, for their valuable comments and suggestions regarding the revision of this thesis, as well as their time and effort.

I would also like to acknowledge my fellow students, Nouman Azam and Yan Zhang, for their constructive suggestions on how to improve academic writing skills and their ways on how to treat people well. Finally, special thanks to the faculty and staffs of the computer science department as well as the Faculty of Graduate Studies and Research. I always find them supportive and helpful during my study. 


\section{Post-Defense Acknowledgements}

I would sincerely appreciate the external examiner, Dr. Yasser Morgan, for his inestimable comments on my thesis. My thanks also go to Dr. Chun-Hua Guo, who is the chair of the defense. 


\section{Dedication}

I wish to give great thanks and appreciation to my wife for her patience, sacrifice, and support during my study and writing of this thesis. Thank-you to my parents and family members for consistent and continous encouragement and contributions to the family. 


\section{Contents}

Abstract $\quad$ i

Acknowledgments $\quad$ ii

Post-Defense Acknowledgments iii

Dedication $\quad$ iv

Table of Contents $\quad$ v

List of Tables $\quad$ ix

List of Figures $\quad$ x

$\begin{array}{lll}\text { Chapter } 1 & \text { Introduction } & 1\end{array}$

$\begin{array}{lll}\text { Chapter } 2 \quad \text { Background } & 4\end{array}$

2.1 Web-based Learning Systems for Computer Science

Subjects . . . . . . . . . . . . . . . . . . . 4

2.1.1 Web-based Learning ............... 5

2.1.2 Review of Web-based Learning Systems for Computer Science

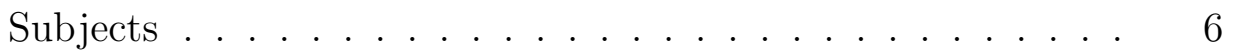


2.1 .3 Limitations . . . . . . . . . . . . . . . . . . 9

2.2 Rough Sets . . . . . . . . . . . . . . . . . . 9

2.2.1 Standard Rough Set Model . . . . . . . . . . . . . . . . . 9

2.2.2 Probabilistic Rough Set Model . . . . . . . . . . . . . . . . 10

2.2 .3 Applications of Rough Sets . . . . . . . . . . . . . . . . . 12

2.3 Web-based Learning Support Systems . . . . . . . . . . . . . . . 12

2.3 .1 What are WLSS . . . . . . . . . . . . . . . 13

2.3 .2 Functions of WLSS . . . . . . . . . . . . . . . . 13

2.3.3 An Architecture of WLSS . . . . . . . . . . . . . . . 14

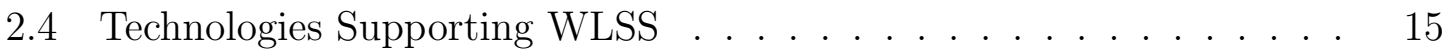

2.4.1 Constraint Satisfaction Problem for Formulating ORS . . . . . 16

2.4.2 Cluster Analysis for Grouping Learners . . . . . . . . . . 16

2.4.3 Moving Average Analysis for Time Series Data . . . . . . . 17

2.5 A Student-centered Learning Style for Adults . . . . . . . . . . . 18

$\begin{array}{lll}\text { Chapter } 3 & \text { Online Rough Sets Development } & 19\end{array}$

3.1 Learning Materials for Rough Sets . . . . . . . . . . . . . . . . . 19

3.1.1 Concepts of Rough Sets _. . . . . . . . . . . . . . . 19

3.1.2 Dependency among Concepts . . . . . . . . . . . . 22

3.1 .3 Quizzes of Concepts . . . . . . . . . . . . . . 23

3.2 Requirement Analysis . . . . . . . . . . . . . . . . . . . . 24

3.2 .1 A Learning Model of ORS . . . . . . . . . . . . . . . . 24

3.2 .2 User Groups . . . . . . . . . . . . . . . . . . . . . . 27

3.2.3 Representing the Learning Model . . . . . . . . . . . . 28 
3.3 System Design . . . . . . . . . . . . . . . . . . . . . 35

3.3 .1 Technical Architecture . . . . . . . . . . . . 35

3.3 .2 Functional Architecture . . . . . . . . . . . . . 37

3.3.3 Design Patterns for Organizing Classes . . . . . . . . . . 39

3.4 Implementation . . . . . . . . . . . . . . . . . 42

3.4.1 Algorithms for Learning Sequences . . . . . . . . . . . . 43

3.4 .2 A Mechanism for Estimated Time . . . . . . . . . . . 47

3.4.3 Programming Languages and Software Environment . . . . . . 48

3.4.4 Implementation of the Expert Component . . . . . . . . . 49

3.4.5 Implementation of the Tutor Component . . . . . . . . . 52

3.4.6 Implementation of the Student Component . . . . . . . . . 55

$\begin{array}{lll}\text { Chapter } 4 & \text { Demonstrative Examples } & 61\end{array}$

4.1 Adaptation in the Whole Learning Process . . . . . . . . . . . . . 61

4.1.1 Planning with Preference and Estimated Time . . . . . . . . 61

4.1.2 Learning Sequences Adapted to Performance . . . . . . . . . . 63

4.2 Adaptation to Preference Containing Multiple

Characters ........................... 64

4.2.1 Defining Multiple Characters as Preference . . . . . . . . . 65

4.2 .2 Analysis of Learning Sequences _. . . . . . . . . . 68

4.3 Comparing ORS with Existing Systems . . . . . . . . . . . . 69

$\begin{array}{lll}\text { Chapter } 5 & \text { Conclusion } & 71\end{array}$

5.1 Concluding Remarks . . . . . . . . . . . . . . . . 71

5.2 Summary of Contributions . . . . . . . . . . . . . 72 
5.3 Future Research . . . . . . . . . . . . . . . . . . . . . . 74

References 


\section{List of Tables}

2.1 Application scope of Web-based learning . . . . . . . . . . . 5

3.1 Concepts of rough sets . . . . . . . . . . . . . . . 21

3.2 Part of the dependency among concepts of rough sets . . . . . . . . . 22

3.3 Open source softwares used in ORS . . . . . . . . . . . . 49

4.1 A study plan including a learning sequence and estimated time . . . . 63

4.2 A study plan adapted to preference containing multiple characters . . 67

4.3 Comparing ORS with existing systems for computer science subjects $\quad 70$ 


\section{List of Figures}

2.1 An architecture of Web-based learning support systems . . . . . . . . 15

3.1 A learning model of ORS . . . . . . . . . . . . . . 25

3.2 Relationships among users of ORS . . . . . . . . . . . 28

3.3 Technical architecture of ORS . . . . . . . . . . . 36

3.4 Functional architecture of ORS . . . . . . . . . . . . . 38

3.5 An adaptor pattern for restricting cross-layer accessibility . . . . . . . 40

3.6 A facade pattern for encapsulating complex business logic . . . . . . . 42

3.7 Predict the estimated time for a new learner A . . . . . . . . . . . 47

3.8 View a list of characters . . . . . . . . . . . . . 50

3.9 Edit details of a character . . . . . . . . . . . . 50

3.10 Edit a concept . . . . . . . . . . . . . . . . 51

3.11 View a list of dependency . . . . . . . . . . . . . 52

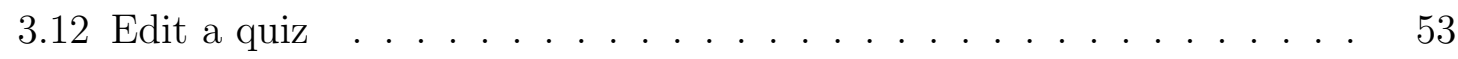

3.13 Define a range from concepts . . . . . . . . . . . . . . 53

3.14 Popup message for recording actual study time of students . . . . . . 54

3.15 Home page of $\mathrm{ORS} \ldots \ldots \ldots \ldots$. . . . . . . . . . 54

3.16 Set a goal from two ways . . . . . . . . . . . . . 55

3.17 Concepts of a goal . . . . . . . . . . . . . . 55 
3.18 Mark concepts as experience . . . . . . . . . . . . . 56

3.19 A goal with experience . . . . . . . . . . . . . 56

3.20 Priority among characters $\ldots \ldots \ldots \ldots \ldots \ldots$

3.21 Priority of character values . . . . . . . . . . . . . 57

3.22 A study plan containing the learning sequence and estimate time . . . 58

3.23 Learn and test a concept . . . . . . . . . . . . . . . . 59

3.24 Notification of a failed quiz . . . . . . . . . . . . . . . . 59

3.25 Progress showing the finished part and unfinished part to a student . 60

4.1 Effect of experience and preference on a goal . . . . . . . . . . 62

4.2 Learning progress reflecting how many concepts are mastered and left 63

4.3 Adaptation to performance leading to the increase of unfinished part 64

4.4 Related concepts of DTRS and Reduct using DM . . . . . . 65

4.5 Preference containing multiple characters . . . . . . . . 66 


\section{Chapter 1}

\section{Introduction}

The Internet has arisen as a main, and perhaps finally the major global communication channel for information and services [12]. It has boosted advances in computer technologies, especially the World Wide Web technology (the Web). The Web offers a distributed infrastructure for information processing and user-friendly interfaces without geographic restrictions [62]. Its wide application and advantages lead to the result that the educational paradigm has been transformed from the teacher-centered model to the student-centered model [16].

Web-based learning is obtaining popularity and provides many benefits compared with traditional learning environments $[15,71]$. It is learning via electronic media on the Web [7]. Web-based learning is an alternative name of e-learning when the delivery channel (i.e., the Web) is emphasized [52]. Its learning content may consist of various forms of multimedia such as text, graphics, audio, video, and animation [46]. Web-based learning extends opportunities for education [27].

In post-secondary education, there are some Web-based learning systems for computer science subjects, such as, artificial intelligence, network security, information 
retrieval, theory of computation, robotic, and system analysis $[8,21,22,26,33,34$, 48, 49]. However, there are no Web-based learning systems for rough sets in the literature.

Rough set theory has gained significant attention as an intelligent tool dealing with uncertainty [3]. It is a mathematical approach to represent and reason uncertain data [72]. The fundamental idea of rough sets is the lower and upper approximations of a set [63]. Based on the approximations, the universe is divided into three regions, namely, the positive, boundary, and negative regions. A major advantage of rough sets is that rules extracted from the three regions can lead to application of the notion of three-way decision [67]. Rough set theory is also quite helpful when trying to find out important features and minimization of conditional attributes [59].

The aim of this dissertation is to design and prototype an effective Web-based learning system for rough sets. Web-based learning support systems (WLSS) provide a reasonable framework to realize Web-based learning. The WLSS sustain teaching as well as learning by making use of the Web technology, and offer learning environments which can be accessed by learners regardless of their time and places [61]. Learners are able to take courses in a Web-based learning support system which contains topics of subjects. Currently, there are some available WLSS to help educators and their learners in education [20, 29]. Moreover, well-designed WLSS can supply studentcentered education [30].

A student-centered learning approach for adults, andragogy, may be suitable for rough sets learners, as most of them are adults. Andragogy aims to help adult learners according to the nature and characteristics of adults [43]. This approach is driven more by needs, experience, preference, and performance of students rather than those 
of teachers.

In this thesis, we present a Web-based learning support system named Online Rough Sets (ORS). We combine WLSS with andragogy to support education, identify a set of rough sets concepts including their dependency information as structured learning materials, and collect a set of concept-specific quizzes. The design of ORS mainly depends on a learning model which is the realization of the learning process of andragogy. The learning model has the mechanism to generate adapted learning materials for each student. With this model, learning materials are tailored and sorted according to each student's preference. Learning materials delivered to a student will be automatically adjusted for review purpose when the student achieves poor performance on any quiz towards a learning material. Moreover, the ORS helps students schedule learning paces and improve learning efficiency by providing estimated learning time for each concept. The estimated learning time can help students reduce interruption caused by time shortage in learning a concept.

The thesis is organized as follows: Chapter 2 elaborates some background about Web-based learning systems in computer science subjects, rough sets, WLSS, technologies supporting WLSS, and andragogy; Chapter 3 describes the development of ORS including learning materials for rough sets, requirement analysis, system design, and implementation. The requirement analysis offers a proposed learning model, user groups, and representation of the model; Chapter 4 presents two demonstrative examples about how to learn rough sets using ORS as well as a comparison between ORS and existing systems; and Chapter 5 gives concluding remarks, summary of contributions, and future research. 


\section{Chapter 2}

\section{Background}

This chapter offers some background and technologies to which this thesis relates. Firstly, we present related works in Web-based learning systems. Secondly, the notion of rough sets is introduced. Subsequently, we give the concept of Web-based learning support systems and its supporting technologies. Finally, a student-centered learning style for adults is introduced.

\subsection{Web-based Learning Systems for Computer Science Subjects}

In this section, we firstly introduce Web-based learning. Subsequently, some Webbased learning systems for computer science subjects are reviewed, and their limitations are highlighted. These systems aim to apply Web-based learning in higher

education especially at the graduate level. Typical subjects include theory of computation, artificial intelligence, robotic, network security, system analysis, and information retrieval. 


\subsubsection{Web-based Learning}

Web-based learning is synonymous with e-learning in some situations.

E-learning refers to the use of electronic media with information and communication technology in education [52]. It is learning through electronic means [36]. The content of e-learning may consist of various forms of multimedia such as text, graphics, audio, video, and animation [46].

E-learning is broadly synonymous with Web-based learning, Web-based training, multimedia learning, computer-based instruction, computer-based training, computeraided instruction, internet-based training, online education, online learning, virtual learning environments, and m-learning [38]. Each of these alternative names emphasizes a particular aspect, component or delivery channel [52].

Web-based learning is used to represent e-learning when the delivery channel (i.e., the Web) is emphasized $[7,50]$. It eliminates the barriers of time and geographical distance in education [27]. Students can participate in their online courses when the Internet is available regardless of their physical locations [27]. Web-based learning also allows students to take courses in a cost effective platform [15]. It offers a second chance to many people for educating themselves [8].

As shown in Table 2.1, Web-based learning can be applied to different sectors and phases in education. These areas include $\mathrm{k}-12$, higher education, and corporate.

Table 2.1: Application scope of Web-based learning

\begin{tabular}{|l|l|}
\hline \multicolumn{1}{|c|}{ Scope } & \multicolumn{1}{c|}{ Description } \\
\hline K-12 & Kindergarten, elementary, junior high, and senior high [44] \\
\hline Higher education & Post-secondary including undergraduate and graduate [54] \\
\hline Corporate & Career training for employees or customers of companies [45] \\
\hline
\end{tabular}




\subsubsection{Review of Web-based Learning Systems for Computer Science Subjects}

There are some Web-based learning systems for computer science subjects reported in the literature. Seven systems are reviewed in this section. Each of these systems aims to support the learning of one subject, with a total of six subjects in computer science involved. We discuss each system in detail and highlight its advantages and disadvantages. The subjects discussed are introduced in the order of theory of computation, artificial intelligence, robotic, network security, system analysis, and information retrieval.

For theory of computation, a weak ontology is used to organize learning materials in an undergraduate program [34]. Concepts related to automata and formal languages have been organized into a hierarchy. The advantage is that the ontology may be used to personalize learning materials based on inference rules and learners' input. The disadvantage is that the ontology only contains the is-a relation among concepts in the hierarchy. In other words, a concept must have and only have one parent concept. Moreover, architectures and implementation of the system are not mentioned.

In another system for theory of computation, a robot is utilized to simulate the details of finite state automata [21]. The system consists of a finite state automaton simulator and a robot. Learners are required to build their own automata graphically in the simulator interface and pass them to the robot. The robot can simulate the automaton transitions. Learners study by observing motions of the robot and playing an off-system game. In the game, a group of learners guess their mutual 
input to strengthen their understanding after observing motions of the robot. The advantage is that details of each automaton are remotely displayed by a real robot. The disadvantage is that the simulator only covers one specific topic of the course, and the game is not a part of the system.

For artificial intelligence, an interface is developed to manipulate input and output of prolog-programmed scripts through the Web [33]. The interface is a web page which offers input options, interprets the options, validates input, and prepares the input to students' prolog predicates. On the output side, the interface explains and formats the output from students' scripts. The advantage is that learners can run their scripts through the Web without installing compile environments on their own computers. The disadvantage is that it doesn't support any other functions.

For robotic, a simulator connecting with a real robot is built to perform experiments [26]. The system aims to ease the difficulty for students to perform actual experiments due to the lack of real robots. The system consists of multimedia contents, a 3D robot simulator, and a real robot. The 16 weeks contents comprises moving pictures, animation, and synthesized voice. The advantage is that a learner can remotely experiment with both a virtual robot and a real one. The disadvantage is that learning materials are fixed and no assessment is mentioned.

For cryptography in network security, the system generates adapted learning materials after a student querying on a particular concept [49]. It contains a set of concepts in cryptography domain and their dependent relationships as an ontology. Each student is required to choose a concept and depth of dependency before querying. The system generates a sequence of concepts after a student querying. The sequence adapts to difficulty based on the learning history of that student, and guides the 
student to learn concepts in this sequence. Related assessment is conducted after learning. The advantage is that learning materials can adapt to student preference and learning history. The disadvantage is that a student can't query on multiple concepts per time, and sequences can't adapt to performance.

For system analysis, a 3D role-play game supports students to learn the waterfall development model by themselves $[8,48]$. The system is a game-based self-learning environment used to assist teaching in an undergraduate course. It also assesses student motivation, satisfaction and learning achievements. The game story is set in a practical office environment, and includes several scenes corresponding to different roles. In each scene, learners study the knowledge in a specific role by viewing goals, executing tasks, and accepting assessment. The advantage is that the game of the system is attractive. The disadvantage is that adaptation to assessment is not considered.

For fuzzy information retrieval systems, a simulator is developed to visualize the steps of weighted queries in a graduate course [22]. It aims to solve the problem that a blackboard is very difficult to visualize the content of weighted queries and fuzzy connectives. The system offers students the opportunity to see and compare the results of different weighted queries. It provides visualization tools to better show evaluation processes of queries step by step. The advantage is that each step can be reviewed clearly. The disadvantage is that the system is just an educational tool for better presentation. 


\subsubsection{Limitations}

Although these learning systems have various advantages, there are still some limitations. We list a few common ones in the following: (1) no adaptation to performance is made; (2) no systems offer queries on multiple concepts; (3) learning materials of systems are organized in a fixed manner except the system for cryptography in network security; and (4) some systems are only simulators or interfaces for the purpose of displaying steps clearly.

\subsection{Rough Sets}

Rough set theory was proposed by Pawlak in 1980s [72]. This section firstly presents two important models of rough sets, namely, the standard rough set model and the probabilistic rough set model. Subsequently, some applications based on rough sets are introduced.

\subsubsection{Standard Rough Set Model}

For the lower and upper approximations, their definitions rely on two extreme cases considering the relationships between a set and a series of equivalence classes [66]. Suppose $U$ is a non-empty finite set of objects, called the universe $U$. Let $E$ be an equivalence (indiscernibility) relation on $U$, i.e., $E \subseteq U \times U$ is reflexive, symmetric, and transitive. The equivalence class of $E$ containing an object $x \in U$ is denoted as $[x]_{E}=[x]=\{y \in U \mid x E y\}$. The family of equivalence classes defined by $E$ is $U / E=\left\{[x]_{E} \mid x \in U\right\}$, and is also called a partition induced by $E$.

Suppose $C$ is a subset of $U$, called a concept. The lower and upper approximations 
of $C$ are defined as below:

$$
\begin{aligned}
& \underline{\operatorname{apr}}(C)=\left\{x \in U \mid[x]_{E} \subseteq C\right\}, \\
& \overline{\operatorname{apr}}(C)=\left\{x \in U \mid[x]_{E} \cap C \neq \emptyset\right\} .
\end{aligned}
$$

The three regions are defined as below:

$$
\begin{aligned}
\operatorname{POS}(C) & =\underline{\operatorname{apr}}(C), \\
\operatorname{NEG}(C) & =U-\overline{\operatorname{apr}}(C), \\
\operatorname{BND}(C) & =\overline{\operatorname{apr}}(C)-\underline{\operatorname{apr}}(C) .
\end{aligned}
$$

The positive region $\operatorname{POS}(C)$ contains those equivalence classes which definitely belong to $C$. In contrast, the negative region $\operatorname{NEG}(C)$ contains those equivalence classes which definitely don't belong to $C$. For each equivalent class in the boundary region $\operatorname{BND}(C)$, one part of its objects belong to $C$, while another part not.

The division of three regions using the standard rough set model is too strict [68]. The degree of overlap between an equivalence class and a concept is not considered. The boundary region is relatively large, while the positive and negative regions are relatively small [68]. This causes unnecessary limitations in application, and many researchers investigate the probabilistic rough set model to ease such limitations [66].

\subsubsection{Probabilistic Rough Set Model}

The probabilistic rough set model utilizes a pair of thresholds $(\alpha, \beta)$ to divide the three regions [66]. The probabilistic association between an equivalence class and 
a concept $C$ is compared with the thresholds to determine the three regions. The probabilistic association is the basis of the model [42] and is shown as follows:

$$
\operatorname{Pr}(C \mid[x])=\frac{|C \cap[x]|}{|[x]|}
$$

where $|\cdot|$ denotes the cardinality of the inside set, $[x]$ is the equivalence class, and $C$ is a subset of the universe. The probabilistic association $\operatorname{Pr}(C \mid[x])$ implies the conditional probability of an object $x \in C$, given that the object $x \in[x]$.

The lower and upper approximations of $C$ divided by the pair are:

$$
\begin{aligned}
& \underline{a p r}_{(\alpha, \beta)}(C)=\{x \in U \mid \operatorname{Pr}(C \mid[x]) \geq \alpha\}, \\
& \overline{\operatorname{apr}}_{(\alpha, \beta)}(C)=\{x \in U \mid \operatorname{Pr}(C \mid[x])>\beta\} .
\end{aligned}
$$

The three regions are defined as below:

$$
\begin{aligned}
& \operatorname{POS}_{(\alpha, \beta)}(C)=\{x \in U \mid \operatorname{Pr}(C \mid[x]) \geq \alpha\}, \\
& \operatorname{BND}_{(\alpha, \beta)}(C)=\{x \in U \mid \beta<\operatorname{Pr}(C \mid[x])<\alpha\}, \\
& \operatorname{NEG}_{(\alpha, \beta)}(C)=\{x \in U \mid \operatorname{Pr}(C \mid[x]) \leq \beta\} .
\end{aligned}
$$

The three regions can be more practical compared with those based on the standard rough set model. The degree of overlap between an equivalence class and a concept is considered. By adjusting the thresholds, the model allows the boundary region to become smaller, while the positive and negative regions may become larger. Larger positive and negative regions are helpful to make decision [70]. 
Based on the probabilistic rough set model, recent development of rough sets includes some models, such as the decision-theoretic rough set model [65, 67], the gametheoretic rough set model [60], and the information-theoretic rough set model [11]. These models differ in how to calculate the pair of thresholds. With such variety, rough sets may be applied in more areas.

\subsubsection{Applications of Rough Sets}

In the field of decision making, the decision-theoretic rough set model is utilized to make three-way decision especially when a nondeterministic situation is meaningful and practical [69]. The game-theoretic rough set model is incorporated to a Webbased medical decision support system in order to perfect the three-way decision making [58].

In the field of data mining, the game-theoretic rough set model is used to conduct feature selection and attribute classification [1,2]. Support vector technologies are extended by rough sets for classification, prediction, and clustering [35].

In the field of education, a system can predict student performance by reduct and rule induction based on the standard rough set model [14].

\subsection{Web-based Learning Support Systems}

This section presents the basic idea, functions, and an architecture of Web-based learning support systems. 


\subsubsection{What are WLSS}

Web-based learning support systems is a specific category of Web-based support systems [15]. Web-based support systems (WSS) refers to a new multidisciplinary research area which studies the Web as the interface, repository and resource center to support human activities [57].

Learning is a dynamic and interactive feedback process in exploring an external environment, and this process is always accompanied with active and imagination driven actions [41]. There are two main learning styles: teacher-centered learning and student-centered learning [15]. Teacher-centered learning is always used in a traditional classroom. Teachers and students communicate bidirectionally in real time. In the student-centered education, students choose what and how they will learn individually, and their abilities, needs, and learning styles are focused [40]. Students are required to be active and responsible participants in their own learning, while teachers or instructors play as supporters or facilitators of learning [15]. This learning style benefits students with personal flexibility.

The WLSS are combination of WSS and learning. The WLSS support teaching and learning by using the Web as an interface, repository, and resource center [30]. Well-designed WLSS are able to offer a student-centered form of education [30].

\subsubsection{Functions of WLSS}

Fan suggests a Web-based learning support system should consider four major functions, namely, the complexity of learning support, the adaptability support, the interaction support, and the assessment support [14]. This conversely means that a 
system can be categorized into WLSS if it can offer those functions through the Web.

The complexity of learning support considers how many actions in the learning process can be supported. The learning process should involve multiple actions. For example, many humans like to interact and collaborate. Experience and obtained knowledge are used in the new cognition. Examples help students consolidate and strengthen their skills. Without these activities, the knowledge presented is always fixed and hard to learn.

The adaptability support means that the system can adapt to each student by considering his/her ability and skill level. The learning materials of instruction, the sequence of topics, the difficulty of topics, and other parameters of instruction are adaptive to each student's experience and performance.

The interaction support enables students to communicate with the system bidirectionally. Learners should be able to store customized annotations and notes to the learning materials. It should allow learners to ask questions and retrieving correct answers in a short period.

The assessment support offers interfaces to evaluate student performance. Testing occupies an important role in evaluating and assessing student progress as well as the check point analysis. Exercises are used to enhance student understanding of each topic through practice. The system should offer each student the opportunity to practice and get according feedback.

\subsubsection{An Architecture of WLSS}

Since WLSS is a specific category of WSS, the general architecture of WLSS inherits some features of WSS, such as the multi-layer design and Web-based user 


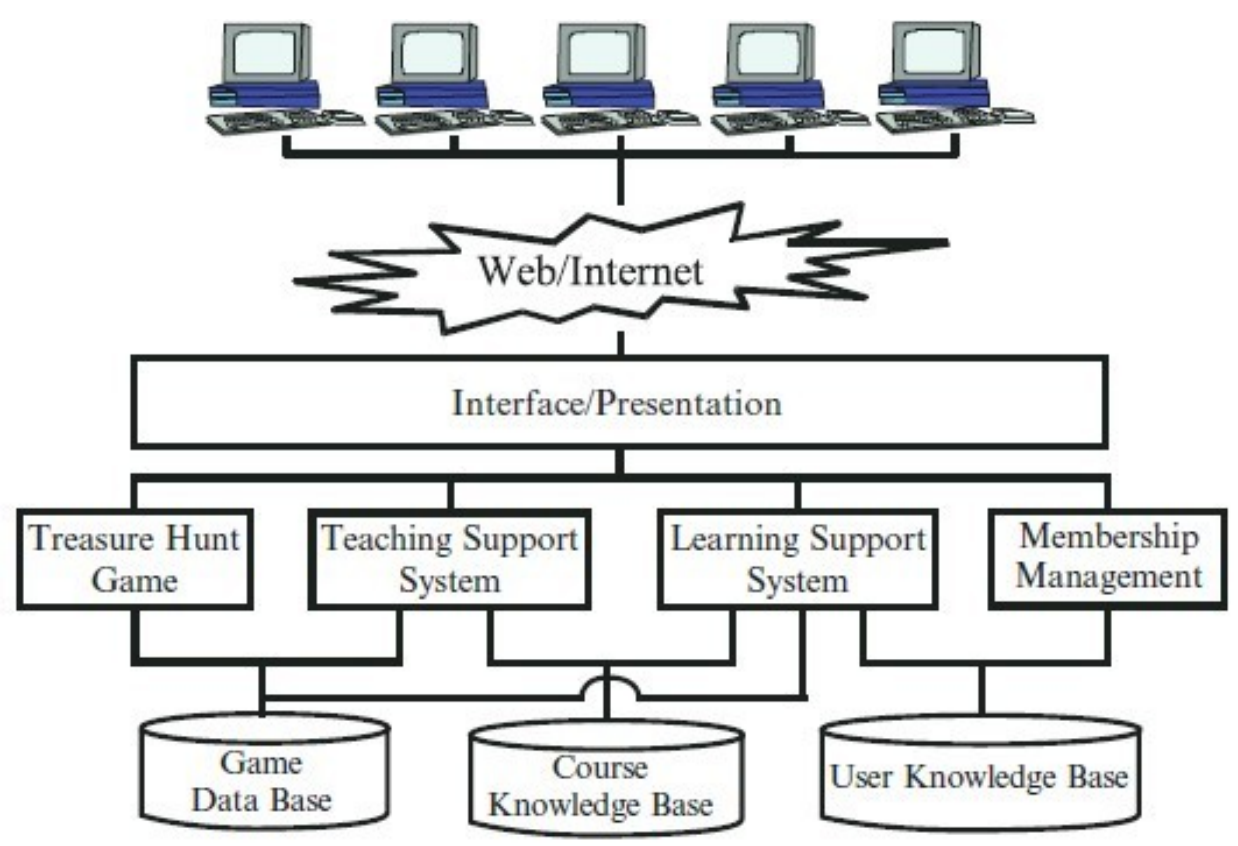

Figure 2.1: An architecture of Web-based learning support systems

interfaces. Architectures of WLSS may vary depending on functions they emphasize, such as the assessment support.

Figure 2.1 shows a demonstrative architecture of WLSS [30]. Based on a treasure hunt game, the system implements the idea of inquiry-based learning to boost student motivation for the purpose of improving effectiveness. The teaching support subsystem enables instructors to design different inquiry-based learning environments according to students. The learning support subsystem sustains inquiry activities of students. The treasure hunt game allows students to study new knowledge, develop thoughts, and review their achievements [30].

\subsection{Technologies Supporting WLSS}

In this section, we present the basic notions of some import technologies used in developing Online Rough Sets. 


\subsubsection{Constraint Satisfaction Problem for Formulating ORS}

In this thesis, we use a constraint satisfaction problem (CSP) to represent the problem of how to generate a personalized learning sequence for a learner. Lots of problems in computer science and artificial intelligence can be treated as special cases of the CSP, such as scheduling, graph problems, circuit design, and temporal reasoning [47]. We treat the generation of a learning sequence as a scheduling problem, and use a CSP to represent and solve it.

In artificial intelligence, a CSP is defined by a set of variables $\left\{x_{1}, x_{2}, \ldots, x_{n}\right\}$ and a set of constraints $\left\{c_{1}, c_{2}, \ldots, c_{m}\right\}$ [47]. Each variable $x_{i}$ has a nonempty domain $D_{i}$ of possible values. Each constraint $c_{i}$ involves a subset of variables and specifies the allowable combinations of values for this subset. There are two types of constraints, namely, hard constraints and soft constraints.

Hard constraints are physical characters which have to be necessarily satisfied, such as spatial and temporal constraints. Soft constraints are properties which should be satisfied as best they can after the hard constraints being met, such as due dates, user preference, and cost [17]. Soft constraints are simply ignored sometimes.

The solution is an assignment of values to some or all of the variables such as $\left\{x_{i}=v_{i}, x_{j}=v_{j}, \ldots\right\}$. Various searching methods can be used to find a solution, such as the depth-first search.

\subsubsection{Cluster Analysis for Grouping Learners}

In this thesis, cluster analysis supports to calculate the estimated time. Cluster analysis (or called clustering) is the job of categorizing a set of objects. Objects 
inside a same group (named a cluster) are more alike with each other, than with those in other groups (clusters excluding the previous cluster). Among the clusters found by different algorithms, objects vary obviously on their inherent characters. In exploratory data mining, cluster analysis is considered as a major job [13].

Various algorithms can be used to conduct cluster analysis. They differ apparently in the concept of what consists a cluster and how to locate them with high efficiency [56]. Some typical algorithms include K-Means, Expectation-Maximization, DBSCAN, and hierarchical clustering [53].

\subsubsection{Moving Average Analysis for Time Series Data}

In this thesis, a moving average supports to calculate the estimated time of each concept. In statistics, a moving average is a type of filter offering finite impulse response. It creates a series of averages from different subsets of the data set. These averages are used to analyze a set of data points [18]. It is also called a running average, rolling average, rolling mean, moving mean, or sliding temporal average.

A moving average is commonly applied on time series data in order to smooth out fluctuations in short terms and highlight trends in long terms [18]. The thresholds of short-term and long-term depend on the situation. The parameters of the moving average will be set accordingly. Some typical types of the moving average include simple moving average, cumulative moving average, weighted moving average, and exponential moving average [24, 28]. 


\subsection{A Student-centered Learning Style for Adults}

Andragogy is a term to represent the art and science of how to teach adults [37]. In order to introduce andragogy, pedagogy is presented firstly.

\section{Pedagogy: Teacher-centered Style}

Pedagogy literally implies leading children [37]. It is also called a traditional or teacher-centered learning approach. In Europe and America, pedagogy remains as the main format for instruction [23]. It is based on the assumption that learners only need to know what the instructors teach them, and places students in a submissive role [23]. In this case, the teaching and learning mainly depends on instructors.

\section{Andragogy: Student-centered Style}

Andragogy is the opposite to pedagogy, and aims to help adults learn better [32]. It is also called a non-traditional or student-centered learning style. Andragogy is based on six assumptions: (1) adults need to know the reason of learning; (2) experience provides the basis; (3) adults need to participant the planning; (4) the learning is problem-centered; (5) the learning should have immediate relevance to adults; and (6) adults respond better to internal motivator [5, 9].

The learning process of andragogy involves eight steps: (1) preparing learners; (2) establishing a climate; (3) involving learners in mutual planning; (4) diagnosing learning needs; (5) forming learning objectives; (6) designing plans with learners;

(7) carrying out plans; and (8) evaluating outcomes [25]. Andragogy is capable to be integrated in WLSS which has flexibility to enable a student-centered learning style. 


\section{Chapter 3}

\section{Online Rough Sets Development}

This chapter describes the proposed system in different aspects including content of the system, requirement analysis, system design, and implementation.

\subsection{Learning Materials for Rough Sets}

Complex knowledge can be separated into a network of prerequisites [10]. Learners should master relevant prerequisites before moving to another higher level knowledge [10]. As the knowledge of rough sets can be represented by a series of formulas, it is possible to decompose them into a set of concepts with dependency. With notes, assignments, and exams of the "Rough Sets \& Applications" course offered at the University of Regina, it is possible to identify the knowledge as a set of concepts, dependency, and concept-specific quizzes.

\subsubsection{Concepts of Rough Sets}

Hierarchical organizations and structures of complex knowledge can be generated by a strategy named the divide and conquer [64]. Table 3.1 shows the 68 concepts that 
we have identified with the strategy in five groups, namely, the Basic, the General, the Advanced, the Application, and the Others.

The group of Basic contains 24 concepts of rough sets. They are the most basic concepts in the subject. Students need to master those concepts if they want to study more advanced concepts. Typical concepts include Information table, Definable set, Partition of universe, Equivalence class (EC), Three regions of rough sets, and Discernibility matrix (DM).

The group of General contains 18 concepts of the subject. They depend on concepts of the Basic group, and are the prerequisites for understanding the Advanced group. Typical concepts include Degree of overlap, Interpretation of $(\alpha, \beta)$, Attribute reduct, Core-attribute, Reduct-attribute, Deletion-based reduct, and Matrix absorption.

The group of Advanced contains 12 concepts of the subject. They are some complex concepts based on the groups of Basic and General. Students may need to spend over one hour to master one concept of this group. Typical concepts include PRS, DTRS, Relative attribute reduct, and Reduct using DM. The number of concepts in this group may increase significantly in the future.

The group of Application contains 2 concepts, namely, Bayesian theorem on PRS and Bayesian decision procedure on PRS. The two concepts are dependent ones for understanding the Advanced group. They involve how to apply theorems of other subjects on rough sets concepts. For instance, Bayesian decision procedure on PRS is the result of applying Bayesian decision procedure on the concept of $P R S$.

The group of Others contains 14 concepts of other subjects. They are dependent concepts for mastering the Basic and Application groups. Typical concepts include Logic connective, Set operator, Power set, De Morgan's laws, Binary relation (BR), 
Table 3.1: Concepts of rough sets

\begin{tabular}{|c|c|c|c|}
\hline Group & Concept & Group & Concept \\
\hline \multirow{22}{*}{ Basic } & Information table & \multirow{6}{*}{ General } & Deletion-based reduct \\
\hline & Equivalence relation (ER) & & Advantage of relative reduct \\
\hline & Concept in an information table & & Matrix absorption \\
\hline & Definable set & & Addition-deletion-based reduct \\
\hline & Family of definable set & & Addition-based reduct \\
\hline & Partition induced by ER & & Powerset-based reduct \\
\hline & Family of undefinable set & \multirow{12}{*}{ Advanced } & PRS \\
\hline & Property of definable set & & DTRS \\
\hline & Family of concept & & GTRS \\
\hline & Partition of universe & & Development of PRS \\
\hline & Property of ER & & Equivalence relation rule \\
\hline & Approximation on definable set & & Element-based rough sets \\
\hline & Property of definable set 2 & & Granule-based rough sets \\
\hline & Decision table & & Subsystem-based rough sets \\
\hline & Discernibility matrix (DM) & & Relative attribute reduct \\
\hline & Non-equivalence relation & & Reduct \\
\hline & Semantic Meaning of ER & & Duality \\
\hline & Equivalence class (EC) & & Reduct using DM \\
\hline & Description of EC & \multirow{2}{*}{ Application } & Bayesian theorem on PRS \\
\hline & Element of DM & & Bayesian decision procedure on PRS \\
\hline & Property of approximation & \multirow{14}{*}{ Others } & Concept \\
\hline & Three regions of rough sets & & Logic connective \\
\hline \multirow{12}{*}{ General } & Simplification & & Means of formula \\
\hline & Minimum DM & & Logic language \\
\hline & Boolean algebra & & Power set \\
\hline & Degree of overlap & & Set operator \\
\hline & ER based on $\mathrm{BR}$ & & De Morgan's laws \\
\hline & Description 2 of EC & & Lattice \\
\hline & Interpretation of $(\alpha, \beta)$ & & Binary relation $(\mathrm{BR})$ \\
\hline & Attribute reduct & & One-to-one correspondence \\
\hline & Non-reduct-attribute & & Bayesian theorem \\
\hline & Core-attribute & & Probabilistic independence assumption \\
\hline & Reduct-attribute & & Bayesian decision procedure \\
\hline & Monotonicity property & & Nash equilibrium \\
\hline
\end{tabular}


Bayesian theorem, and Probabilistic independence assumption.

Besides, each concept has a description of its notion, a group of detailed formulas, and several examples.

\subsubsection{Dependency among Concepts}

Dependency exists among related concepts. There are 103 dependent relationships among the above 68 Concepts. Each relationship involves a parent concept and a child one. Understanding the parent concept is the prerequisite for learning the child one.

Table 3.2: Part of the dependency among concepts of rough sets

\begin{tabular}{|l|l|}
\hline Prerequisite Concept & \multicolumn{1}{|c|}{ Concept } \\
\hline Set operator & Information table \\
\hline Information table & Equivalence Relation \\
\hline Information table & Logic language \\
\hline Logic connective & Logic language \\
\hline Information table & Means of formula \\
\hline Logic language & Means of formula \\
\hline Concept & Concept in an information table \\
\hline Information table & Concept in an information table \\
\hline Logic language & Concept in an information table \\
\hline Means of formula & Concept in an information table \\
\hline Information table & Definable set \\
\hline Logic language & Definable set \\
\hline Means of formula & Definable set \\
\hline Definable set & Family of definable set \\
\hline Power set & Family of definable set \\
\hline Family of definable set & Approximation on definable set \\
\hline Set operator & Approximation on definable set \\
\hline Family of definable set & Property of definable set \\
\hline Lattice & Power set \\
\hline Equivalence relation & Partition induced by ER \\
\hline Partition of universe & Partition induced by ER \\
\hline Family of definable set & Family of undefinable set \\
\hline Power set & Family of undefinable set \\
\hline
\end{tabular}


In other words, students should not study the child concept if they do not master the parent one. Those relationships make up a hierarchical structure among the concepts.

For illustration, Table 3.2 partly lists dependency among concepts of rough sets. In this table, the column of Prerequisite Concept corresponds to parent concepts, while the column of Concept represents child concepts. For instance, a student needs to master Set operator before studying Information table. Definable set has three prerequisite concepts including Information table, Logic language, and Means of formula. All the three concepts should be mastered by students before learning Definable set.

\subsubsection{Quizzes of Concepts}

Every concept is accompanied with its own quizzes. Mastering a concept means the student has to successfully pass all its quizzes. The types of quizzes include choice selection and fill-in-the-blank.

For a quiz of choice selection, it contains a question and several answers including both right and wrong solutions. For instance, a question of Logic language is "Please select a true one from the following statements, where the domain is all real numbers." The four candidate answers are in Equation (3.1). In this case, the right solutions are (a) and (b), while the wrong solutions are (c) and (d).

$$
\begin{aligned}
& \text { (a) } \exists_{x}\left(x^{4}<x^{2}\right), \\
& \text { (b) } \exists_{x}\left(x^{2}>x\right), \\
& \text { (c) } \forall_{x}\left((-x)^{2}=x^{2}\right), \\
& \text { (d) } \left.\forall_{x}\left((-x)^{2}>x\right)\right\} .
\end{aligned}
$$


For a quiz of fill-in-the-blank, it contains a question together with an improper answer, and students need to complement this answer correctly after reading the question. For instance, a question of Granule-based rough sets is "Please complement the blanks in the formula which represents the granule-based rough sets, given $E$ is an equivalence relation on $U$ and $C \subseteq U$." The improper answer is Equation (3.2), and the right solution is Equation (3.3).

$$
\begin{aligned}
& \underline{\operatorname{apr}}(C)=\cup\{[x] \mid[x] \in U / E,[x] \underline{\text { please fill } C\},} \\
& \overline{\operatorname{apr}}(C)=\cup\{[x] \mid[x] \in U / E,[x] \underline{\text { please fill }} C \underline{\text { please fill }\}} . \\
& \underline{\operatorname{apr}}(C)=\cup\{[x] \mid[x] \in U / E,[x] \subseteq C\}, \\
& \overline{\operatorname{apr}}(C)=\cup\{[x] \mid[x] \in U / E,[x] \cap C \neq \emptyset\} .
\end{aligned}
$$

\subsection{Requirement Analysis}

The aim of ORS is to support students to learn rough sets through the Web in a student-centered learning environment. The learning materials of ORS are a set of concepts with dependency presented in Section 3.1.1, and each concept is accompanied with a set of concept-specific quizzes.

\subsubsection{A Learning Model of ORS}

A learning model of ORS is the realization of the eight-step learning process which is described in Section 2.5. The learning model supports students to study 
those dependent concepts from bottom to top with some personalization. It also enures that prerequisites of a concept must be studied again when learners fail to pass the concept's quizzes [10]. This is done by adding a loop between Forming learning objectives and Evaluating outcomes. Figure 3.1 shows the learning model and its emphasis (i.e., preference, performance, and estimated time).

A concept is a unit of learning materials defined by experts, and is introduced in Section 3.1.1. An item of dependency is a dependent relationship between two concepts which is introduced in Section 3.1.2. A range defined by tutors points to several concepts, and is used to help students choose their learning objectives. A goal indicates several concepts selected by a student from either a concept list or ranges. An item of experience is a group of concepts which appear in a goal and have been

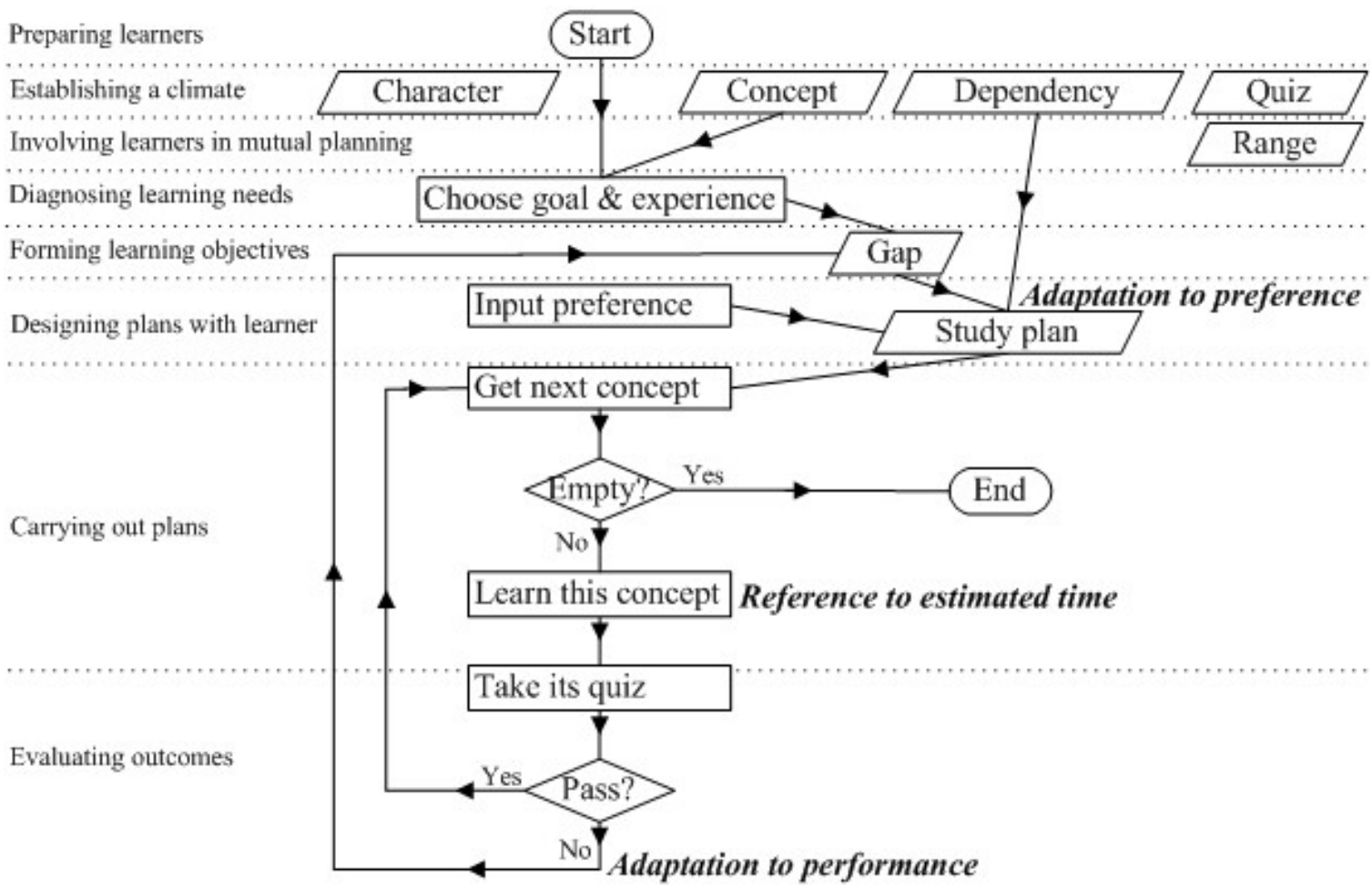

Figure 3.1: A learning model of ORS 
mastered by the student. An item of preference contains prioritized constraints based on concept characters (e.g., the difficulty, the relevance, the sub-subject, the media type), and is used to customize learning sequences for everyone.

Learning materials delivered to each individual are organized in a study plan. The study plan contains a learning sequence and estimated time for learning each concept. The learning sequence guides a student to learn concepts one by one sequentially. The estimated time indicates how long a learner may need to learn each concept. It helps a student allocate proper time to learn a concept, and thus helps the student reduce interruption caused by time shortage in learning this concept. The estimated time can improve learning efficiency, since learners need more time to finish a task in case it has been interrupted [4].

Adaptation of Web-based learning systems focuses on curriculum sequencing to guide students and diagnose their misconceptions through their learning processes [6]. In the above learning model, adaptation to preference means that ORS forms a learning sequence based on dependency and learner preference. Adaptation to performance means that ORS modifies a learning sequence by adding prerequisites of a concept into this sequence when the student achieves poor performance on this concept.

An example is presented to introduce the running of the above learning model. Suppose a student has a goal containing 7 concepts, namely, Set operator, Logic connective, Information table, Logic language, Means of formula, Concept, and Concept in an information table. We also assume his experience contains the first 4 concepts as listed. His gap becomes Means of formula, Concept, and Concept in an information table. The difficulty levels of the difficulty character on the 3 concepts are normal, low, and normal, respectively. 
After the student selecting and configuring the character of difficulty as preference (e.g., from high to low), a study plan is generated based on dependency and preference. It contains a learning sequence of Concept $->$ Means of formula $->$ Concept in an information table. The step of Get next concept results the start of learning Concept. According to the estimated time in the study plan, the student can evaluate whether or not he has enough time to learn this topic for the purpose of reducing interruption caused by time shortage.

Suppose the student decides to learn Concept, and passes its related quizzes. The gap becomes Means of formula and Concept in an information table. Subsequently, the student learns Means of formula but fails on its quizzes. The learning model modifies the gap by adding the prerequisite concepts of Means of formula based on dependency. The gap becomes Logic language, Means of formula, and Concept in an information table.

Based on the new gap, the study plan is updated. The student learns concepts according to the new study plan. In this process, the learning model may increase or decrease the size of gap due to the student's performance. The learning model will end the process after the gap becoming empty.

\subsubsection{User Groups}

There are four groups of users, namely, expert, tutor, student, and administrator. Experts are responsible for defining learning materials, dependency, and their characters. Tutors help students customize these learning materials. Students define their preference and study the personalized learning materials. Administrators manage the menu, roles, and other configuration of the system. 


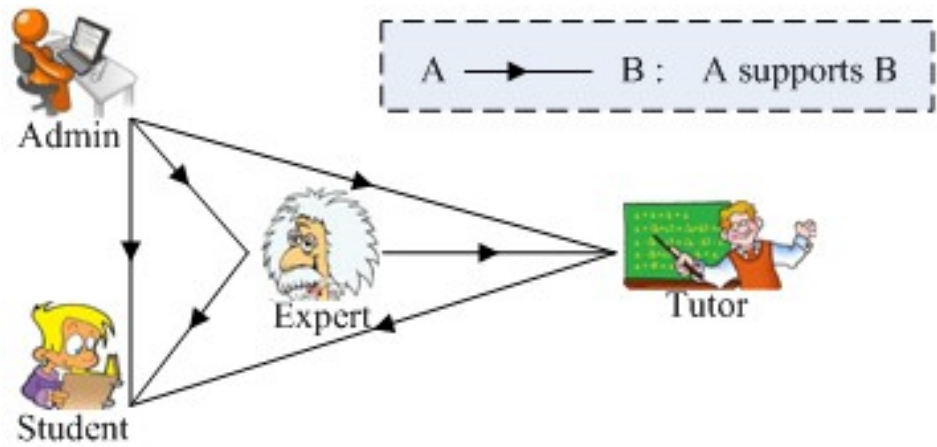

Scenario 1: Lecture

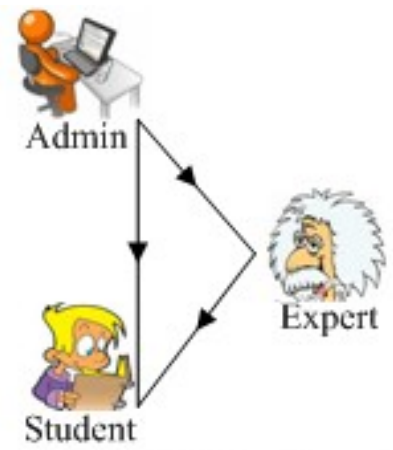

Scenario 2: Individual learning

Figure 3.2: Relationships among users of ORS

Among the four groups of users, their relationships can be expressed in two typical scenarios as shown in Figure 3.2. For all scenarios, experts can define learning materials with support of administrators. Students can use the system with support of experts and administrators.

In scenario 1, a student enrolls a lecture course of rough sets. A tutor (instructor) defines several ranges according to different student expectations. The student is supposed to select an appropriate range according to his situation. For this student, the study on ORS is supplementary to the lecture. In scenario 2, a student learns individually. The student is supposed to choose a few concepts and study them by himself through ORS. A person who wants to learn the subject systematically by himself is also supposed to use ORS in this way.

\subsubsection{Representing the Learning Model}

This section describes the representation of the learning model presented in Section 3.2.1. Topics in this section include concept, knowledge, learning order relationship, partial order, preference, and learning sequence. 


\section{Concept and Knowledge}

Concepts of rough sets are listed in Section 3.1.1. Each concept refers to its name, description, links of learning content, related quizzes, and values of characters. A concept $C$ can be expressed by a tuple:

$$
C=\{i d, N, I, L, Q, P\}
$$

where id is a unique identifier of the concept;

$\mathrm{N}$ is a set of names of the concept;

I is a set of intensions which are description of the concept;

$\mathrm{L}$ is a set of hyper links which connect to the learning content;

$\mathrm{Q}$ is a set of pairs of (question, answers) which are used as quizzes;

$\mathrm{P}$ is a set of pairs of (character, character_value), such as (difficulty, low).

For each concept, it can relate to several characters, and has one and only one value on each character. The time spent on a concept consists of two parts, namely, understanding its intensions and solving its quizzes.

In computer science and information science, the knowledge of a subject (or domain) can be represented as a set of concepts and relationships between pairs of concepts from the perspective of ontology. If a student wants to learn a subject, the student needs to master its concepts and their relationships at least. In this thesis, we define the set of concepts of a subject as $S C=\{C \mid C$ is a concept $\}$. 


\section{Learning Order Relationship among Concepts}

A learning order between two concepts $C 1$ and $C 2$, is called a learning order relationship if and only if learning $C 1$ is a prerequisite for learning $C 2$. It is denoted by:

$$
C 1 \preceq C 2,
$$

where $C 1, C 2 \in S C, C 1$ is called a parent concept, and $C 2$ is called a (child) concept.

The learning order relationship has same meaning with the dependent relationship introduced in Section 3.1.2. In terms of a subject $S C$, its learning order relationships construct a hierarchical structure among its concepts, and can be denoted by:

$$
L O R_{\text {full }}=\{(x, y) \mid x \preceq y, x \neq y, x \in S C, y \in S C\} .
$$

For instance, we assume a subject and its learning order relationships as follows:

$$
\begin{aligned}
& S C=\{C 1, C 2, C 3, C 4, C 5, C 6, C 7\}, \\
& L O R_{\text {full }}=\{(C 3, C 2),(C 2, C 4),(C 1, C 4),(C 4, C 6),(C 5, C 7)\} .
\end{aligned}
$$

In terms of $C 4$, there are $C 1 \preceq C 4$ and $C 2 \preceq C 4$. We can also say that learning $C 1$ and $C 2$ is necessary to learn $C 4$.

The knowledge state means the state of learning. A knowledge state contains a set of concepts, and the prerequisites of these concepts are also included in the set. A subset $K S \subseteq S C$ is called a knowledge state on $S C$, if it satisfies:

$$
\forall_{y \in K S}\left(\neg \exists_{x \in S C}\left((x, y) \in L O R_{f u l l}\right) \bigvee \forall_{(x, y) \in L O R_{f u l l}}(x \in K S)\right)
$$

where $S C$ denotes the set of concepts of a subject; 
$L O R_{f u l l}$ denotes learning order relationships of the subject.

For instance, $\{C 2, C 3, C 5\}$ is a knowledge state in the above example. The subset contains all the prerequisites of its elements.

Not every subset of $S C$ is a knowledge state. For instance, $\{C 2, C 5\}$ is not a knowledge state in the above example. The prerequisite of $C 2$ is $C 3$, but $C 3$ doesn't belong to the subset.

The family of all possible knowledge states is called the knowledge structure $(K S T)$, denoted by:

$$
K S T=\left\{K S \subseteq S C \mid \forall_{y \in K S}\left(\neg \exists_{x \in S C}\left((x, y) \in L O R_{\text {full }}\right) \bigvee \forall_{(x, y) \in L O R_{\text {full }}}(x \in K S)\right)\right\}
$$

We can treat the objectives chosen by a student as an arbitrary subset $A \subseteq S C$. The subset $A$ can be transformed to a knowledge state $K S_{A}$ by:

$$
\begin{aligned}
& \text { BaseCase: } K S_{A}=A \bigcup\left\{x \in S C \mid x \notin A, y \in A,(x, y) \in L O R_{\text {full }}\right\} \\
& \text { Recursive: } K S_{A}=K S_{A} \bigcup\left\{x \in S C \mid x \notin K S_{A}, y \in K S_{A},(x, y) \in L O R_{\text {full }}\right\} .
\end{aligned}
$$

For instance, a subset $A=\{C 2, C 5\}$ is not a knowledge state in the above example. It can be transformed into the knowledge state $K S_{A}=\{C 2, C 3, C 5\}$ by adding $C 3$ into itself. The $C 3$ is the result of searching prerequisites of $A$ according to $L O R_{\text {full }}$.

Suppose subset $O b j \subseteq S C$ contains the objectives selected by a student. The Goal is $K S_{O b j}$. The student needs to learn all concepts in the Goal for the purpose of completing his/her objectives.

Before starting to learn the Goal, the student may have mastered some of its 
concepts. These concepts are unnecessary to be studied again. The Experience represents those concepts, and Experience $\subseteq$ Goal.

As the time as the Experience is marked by the student, a simpler set of learning order relationships $L O R \subseteq L O R_{\text {full }}$ can be denoted by:

$$
\left.L O R=\left\{(x, y) \mid(x, y) \in L O R_{\text {full }} \wedge y \in \text { Goal } \wedge x \notin K S_{\text {Experience }}\right)\right\} .
$$

The Gap is the real learning objectives after considering the student's Goal and Experience. It contains a set of concepts, and is denoted by:

$$
\text { Gap }=\text { Goal } \bigcap \overline{\overline{K S \text { Experience }} .}
$$

\section{Partial Order for A Set of Concepts}

A partial order used here is a linear sequence of concepts in a Gap. With a partial order, the student can learn all the concepts in the Gap one by one without dissatisfying the $L O R$. There may be multiple partial orders for a Gap and its $L O R$. As introduced in Section 2.4.1, we form a constraint satisfaction problem to find out all possible partial orders as follows:

- Variables: $X=\left\{x_{1}, x_{2}, \ldots x_{n}\right\}$, where $n$ is the cardinality of the Gap.

- Domains: $\forall_{x \in X}$, the domain of $x$ is $D_{x}=\{c \mid c \in \operatorname{Gap}\}$.

- Constraints:

1. $x_{i} \neq x_{j}$,

2. $\forall_{(p, q) \in L O R} \neg \exists_{x_{r}, x_{s} \in X}\left(x_{r}=q \bigwedge x_{s}=p \bigwedge 1 \leq r<s \leq n\right)$. 
A partial order is one kind of assignment of values from $D_{x}$ to $x$ for every $x \in X$ without breaking the $L O R$. For a student, concept $x_{i} \in X$ is the $n o . i^{\text {th }}$ step to be studied. The partial order can be expressed as $x_{1}->x_{2}->x_{3}->x_{4}->\ldots->$ $x_{n}$. For example, a student wants to learn a few concepts of rough sets, and the Gap $=$ $\{$ Degree of overlap, Interpretation of $(\alpha, \beta)$, Equivalence class $(E C)$, Approximation on definable set $\}$. One partial order is Equivalence class $(E C)->$ Degree of overlap $->$ Aapproximation on de finable set $->$ Interpretation of $(\alpha, \beta)$.

\section{Student Preference for Customizing Partial Orders}

In a concept $C=\{i d, N, I, L, Q, P\}$, a set $P$ contains several pairs of (character, character_value), such as (difficulty,low). In this case, the difficulty is a character of concepts, while the low is a value of this character.

Let the set of characters be Character $=\{x \mid(x, y) \in P\}$, and the domain of a character $p$ is $D_{p}=\{y \mid(x, y) \in P \bigwedge x=p\}$.

Preference is defined by each student, and is used to find a unique partial order from multiple ones. This partial order should satisfy the student's preference most. An item of preference contains two hierarchical priorities, namely, priority of characters and priority of character_value in each character. Priority of characters is always satisfied firstly. Besides, priority of character_value in each character is considered as best it can. The definition of the preference consists of:

- Pref $=\{(p$, priority $) \mid p \in$ Character $\}$, where $\mid$ Pref $|\leq|$ Character $\mid$. It reflects student preference in the hierarchy of character. $\forall_{(p, \text { priority }) \in \text { Pref }}$, priority defines the learning priority of character $p$ in comparison with other characters in Pref. 
- PrefDetail $l_{p}=\left\{x_{1}, x_{2}, \ldots x_{n}\right\}$, where $n=\left|D_{p}\right|, x_{i} \in D_{p}$, and $x_{i} \neq x_{j}$. It reflects student preference in the hierarchy of character_value in character $p . \forall_{x_{i} \in D_{p}}$, the value of $x_{i}$ defines the learning priority compared with those of others in Pref Detail $p$.

- The semantic meaning of Pref and PrefDetail $p$ is described as follows:

$-S G \subseteq G a p$.

- $S G$ can be divided into $n$ parts by Pref. These parts can be denoted by $S G^{p_{1}}, S G^{p_{2}}, \ldots S G^{p_{n}}$, where $\left|S G^{p_{1}}\right|+\left|S G^{p_{2}}\right|+\ldots\left|S G^{p_{n}}\right|=|S G|$. Concepts of $S G^{p_{i}}(1 \leq i \leq n)$ is the $n o . i^{\text {th }}$ batch to be studied.

- $S G^{p_{i}}$ can be further divided into $m$ parts by Pref Detail $p_{p_{i}}$. These parts can be denoted by $S G_{x_{1}}^{p_{i}}, S G_{x_{2}}^{p_{i}}, \ldots S G_{x_{m}}^{p_{i}}$, where $\left|S G_{x_{1}}^{p_{i}}\right|+\left|S G_{x_{2}}^{p_{i}}\right|+\ldots\left|S G_{x_{m}}^{p_{i}}\right|=$ $\left|S G^{p_{i}}\right|$. Among concepts of $S G^{p_{i}}, S G_{x_{j}}^{p_{i}}$ is the no. $j^{\text {th }}$ batch to be studied given $(1 \leq i \leq m)$.

\section{Learning Sequence for A Set of Concepts}

The learning sequence is a unique partial order which satisfies the preference of a student most. We modify the constraint satisfaction problem as follows:

- Variables: $X=\left\{x_{1}, x_{2}, \ldots x_{n}\right\}$, where $n$ is the cardinality of the Gap.

- Domains: $\forall_{x \in X}$, the domain of $x$ is $D_{x}=\{c \mid c \in$ Gap $\}$.

- Hard constraints:

1. $x_{i} \neq x_{j}$, 
2. $\forall_{(p, q) \in L O R} \neg \exists_{x_{r}, x_{s} \in X}\left(x_{r}=q \bigwedge x_{s}=p \bigwedge 1 \leq r<s \leq n\right)$.

- Soft constraints: Pref and PrefDetail

Variables, domains, and hard constraints of this problem are same with those which are introduced in Partial Order. However, soft constraints are added. They

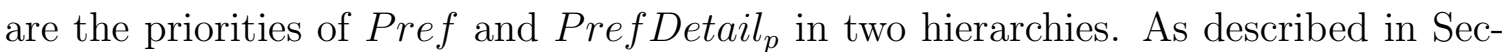
tion 2.4.1, the hard constraints are mandatary to be satisfied, and the soft ones should be met as best they can.

\subsection{System Design}

This section presents the technical architecture, the functional architecture, and design patterns used for organizing classes or objects in ORS.

\subsubsection{Technical Architecture}

A technical architecture describes the separation of components regarding technical issues rather than business logic. Model-view-controller (MVC) has been widely used in designing web applications and conforms to the multi-layer architecture of WLSS [15]. The MVC has evolved to many variations. A passive implementation of MVC neglects notifications between the model component and others (view and controller components), since these notifications are not required or are not supported by software platforms [39]. In the scenario of web applications, a browser is difficult to receive "notify" since it is a classic "pull" mechanism rather than "push". One of such passive implementations is named "Model 2" [51]. 


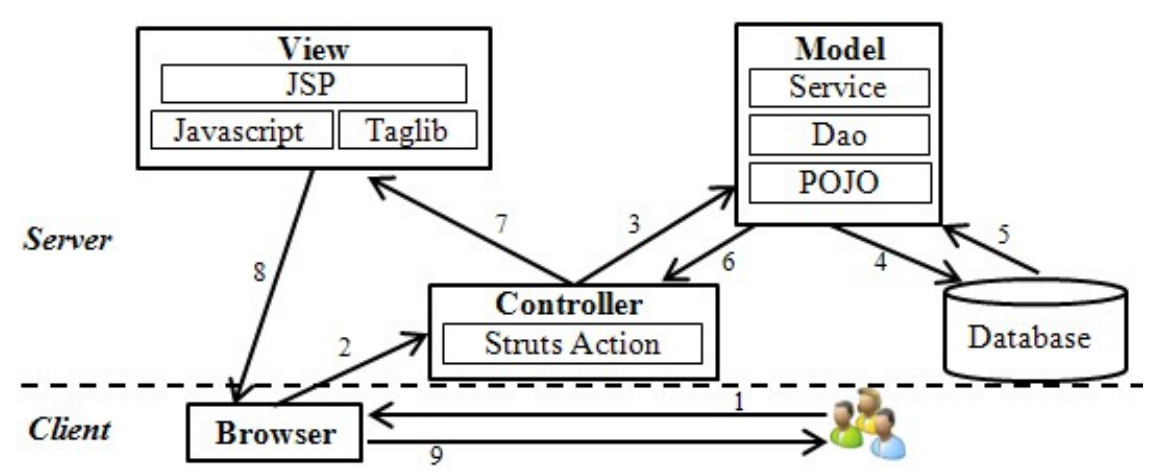

Figure 3.3: Technical architecture of ORS

The technical architecture of ORS is extended from "Model 2" as shown in Figure 3.3. The View generates graphical interfaces to users. The Controller handles the HTTP request and response between users and the system. The Model encapsulates the business logic of ORS. The Model is divided into three layers, namely, the Service, the Dao, and the POJO. Each class of the POJO, or Plain Old Java Object, represents a table in the Database. The Dao, or Data Access Object, contains classes which operate data on a single class of the POJO. The Service encapsulates business logic among multiple classes of the Dao and serves the Controller.

When a user utilizes a browser to access ORS, the data flow proceeds as follows:

Step 1: a user manipulates on the Browser.

Step 2: the Browser issues a HTTP request to the Controller.

Step 3: the Controller receives the request. If its inner business rules are not enough to handle, the Controller sends a request to the Model.

Step 4: the Model sends a query to the Database for that request.

Step 5: the Database responses corresponding data to the Model.

Step 6: the data is processed by complex business rules of the Model. The processed information is sent to the Controller. 
Step 7: the Controller makes decision to choose a specific page in the View.

Step 8: the specific page equipped with related data is sent to the Browser.

Step 9: this page is presented to the user by the Browser.

For each request launched by a user, there may be three modes of data flow, namely, the complete mode, the moderate mode, and the simple mode. The complete mode needs data from the Database and contains all the steps. In the moderate mode, business rules of the Controller and the Model are enough to make decision. There is no need to operate data from the Database. It only involves Steps 1, 2, 3, 6, 7, 8, and 9. In the simple mode, the inner business rules of the Controller is enough to make decision. There is no need to operate data from the Model and the Database. It only involves Steps 1, 2, 7, 8, and 9 .

Since the multi-layer structure and use of browsers conform to the WLSS definition presented in Section 2.3.3, this architecture can be treated as a design of WLSS.

\subsubsection{Functional Architecture}

A functional architecture describes the separation of components regarding business logic rather than technical issues. As shown in Figure 3.4, the functional architecture of ORS includes four main functional components, namely, the Expert area, the Tutor area, the Student area, and the Public management area.

The Expert area is designed to input concepts, dependency, and quizzes by experts. In particular, the Concept is used to create, read, update, and delete (CRUD) a concept, which contains the name, description, links of research papers, quizzes, and values on characters (e.g., the low on the difficulty character). The Dependency CRUD dependent relationships among concepts. The Quiz CRUD a question and 


\begin{tabular}{|c|c|c|c|}
\hline \multirow{2}{*}{\multicolumn{2}{|c|}{$\begin{array}{l}\text { Public management } \\
\text { area }\end{array}$}} & User & Role \\
\hline & & \multicolumn{2}{|c|}{ Configure } \\
\hline \multicolumn{4}{|c|}{ 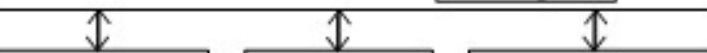 } \\
\hline Expert area & \multicolumn{2}{|c|}{ Tutor area } & Student area \\
\hline \multirow[b]{2}{*}{ Concept } & \multirow{2}{*}{\multicolumn{2}{|c|}{ Range }} & Goal \\
\hline & & & Experience \\
\hline \multirow[b]{2}{*}{ Dependency } & \multirow{2}{*}{\multicolumn{2}{|c|}{\begin{tabular}{|c|} 
Estimated \\
time
\end{tabular}}} & Preference \\
\hline & & & Study plan \\
\hline \multirow{2}{*}{ Quiz } & \multirow{2}{*}{\multicolumn{2}{|c|}{$\begin{array}{c}\text { Record } \\
\text { time }\end{array}$}} & My progress \\
\hline & & & Working place \\
\hline \multirow{2}{*}{ Character } & \multirow{2}{*}{\multicolumn{2}{|c|}{ Archive }} & Modify SP \\
\hline & & & Personality \\
\hline
\end{tabular}

Figure 3.4: Functional architecture of ORS

candidate answers for each concept. The Characters CRUD a character (e.g., the difficulty) and its details (e.g., low, normal, high).

The Tutor area allows human tutors to help learners set up their personal goals, and predicts as well as records student learning time as a virtual tutor. In particular, the Range helps tutors CRUD some predefined objectives, and a learner can select one of them as the learner's goal. The Estimated time predicts how long a learner may spend to master a particular concept. For each concept, this time may be a value or a combination of values coming from the averages of the best $\mathrm{N}$ similar learners, the worst $\mathrm{N}$ similar learners, the latest $\mathrm{N}$ similar learners, etc. The Record time logs the actual learning time spent on each concept. The Archive stores the study history of each learner.

The Student area provides learners the interaction interfaces with ORS. In particular, the Personality is used to maintain student personal information (e.g., age, educational level). The Goal CRUD a goal from ranges or concepts. The Experience allows a student to mark those concepts which he/she has already mastered in his goal initially. The Preference allows students to have some preference (e.g., from low to 
high on the difficulty character) on learning sequences without breaking dependency among concepts. The Study plan generates a linear study plan based on a goal, experience, and preference. The My progress displays how many concepts students have learned and how many remaining concepts. The Working place displays content of a concept and quizzes associated with it. The Modify SP finds out dependent concepts when a student fails in a quiz, sorts them based on preference, then adds them into the old study plan.

The Public management area lets administrators manage the basic configurations of the system. The Configure is used to define the menu, its internal links, and parameters of the Estimated time (such as the N). The Role is used to define how many functions a role could access. User manages user roles and basic information (e.g., contact information).

According to the definition of WLSS in Section 2.3.2, the Expert area contributes to the function of the complexity of learning support, and the Tutor area relates to the Interaction support. The Student area provides functions which are similar with the Adaptability support and the Assessment support. The architecture can be treated as a design of WLSS.

\subsubsection{Design Patterns for Organizing Classes}

Design patterns are used to ease the design of programs in each component of the above architectures. They are refusable and empirical templates which define the structures of classes or objects. The main categories of design patterns are creational, structural, and behavioral patterns. In this thesis, two structural patterns are used to develop some functions of ORS, namely, the adaptor and the facade. 


\section{Adaptor}

An adaptor can help a client program easily access an incompatible class without changing this class and the client [19]. An object is called an adaptor if it satisfies: (1) it provides a method to clients; (2) These clients can not directly access another class for data format or other reasons; (3) The method has a suitable interface to be invoked by those clients; (4) The method encapsulates business logic of invoking that class; and (5) The class (adaptee) is passed into the object as a parameter of the method or a constructor.

One typical application of the adaptor pattern in ORS is the Dao which is introduced in Section 3.3.1. There are three layers in the Model, namely, the Service, the Dao, and the POJO. According to the architectural style, an object in the Controller can only access the Dao in order to get data from the POJO. An adaptor is required

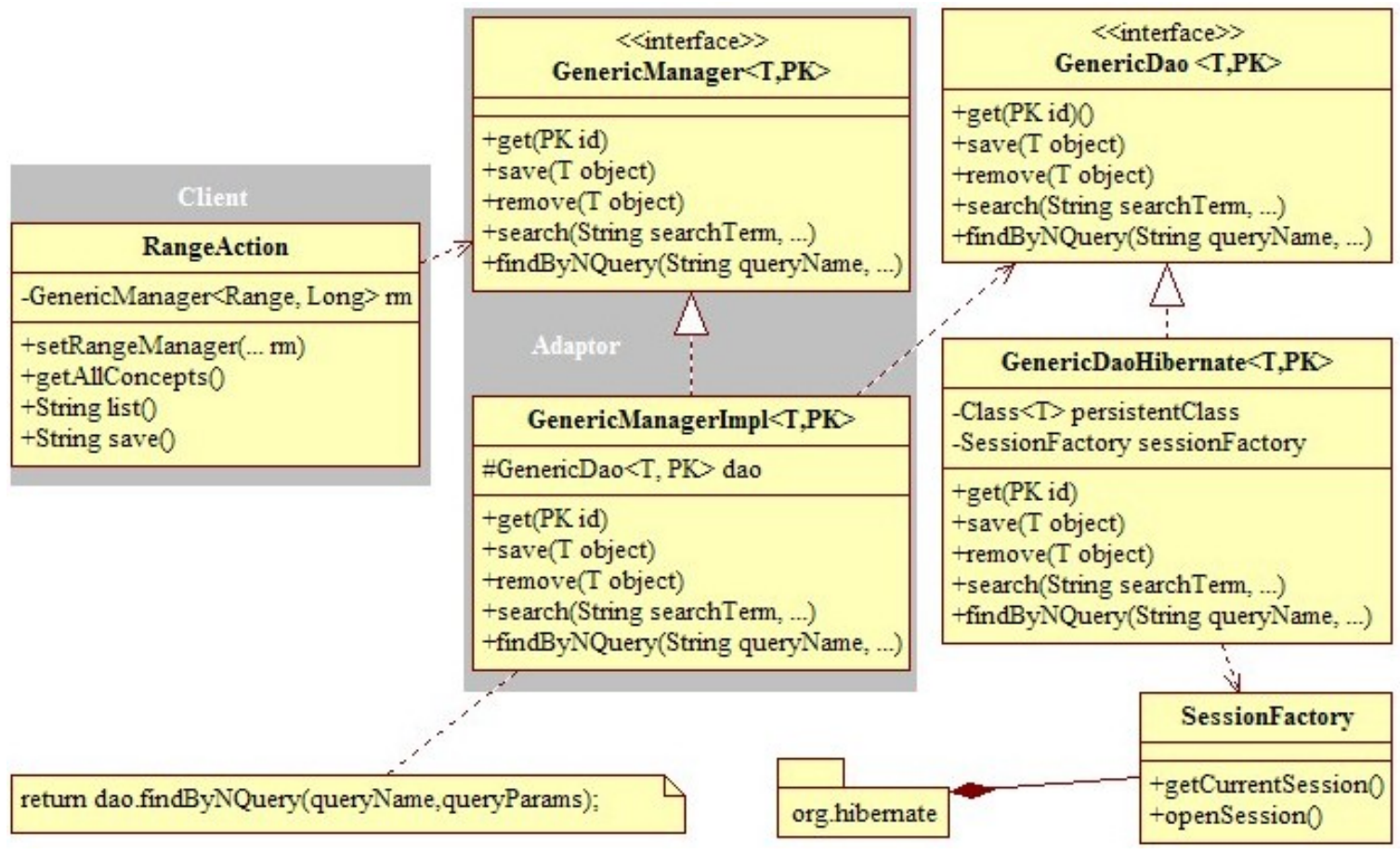

Figure 3.5: An adaptor pattern for restricting cross-layer accessibility 
to satisfy that style.

As shown in Figure 3.5, the client is RangeAction.java and the adaptor is GenericManager.java. The Adaptee is GenericDao.java. Business logic has been encapsulated in methods of the adaptor, such as save( $T$ object). The client is easier to utilize classes in other components. In another case, the adaptor is GenericDao.java if GenericManagerImpl.java tries to access SessionFactory.java.

\section{Facade}

A facade is able to help a client program simply access a series of classes through an interface regardless of the dependency among these classes [19]. An object is called a facade if it satisfies: (1) it provides several methods to clients; (2) each method is a simplified and unified interface to operate a set of classes belonging to a class library or subsystem; and (3) No method can be invoked by those classes.

One typical application of the facade pattern in ORS is the Estimated time which is introduced in Section 3.3.2. This function utilizes a clustering algorithm from the software package, named weka, to find a group of previous users having similar personality with a new user. It involves steps of data preparation, building model, and executing model. Each step may refer to multiple classes and their dependencies of weka and JDBC. A facade is required to ease the manipulation of classes for client programs.

As shown in Figure 3.6, the client is PlanServiceImpl.java and the facade is ClusterImpl.java. Others are supporting classes of weka and JDBC. Complex business logic has been encapsulated in two methods of the facade, namely, the prepareData() and the doCluster(). The client is easier to utilize weka through the two methods. 


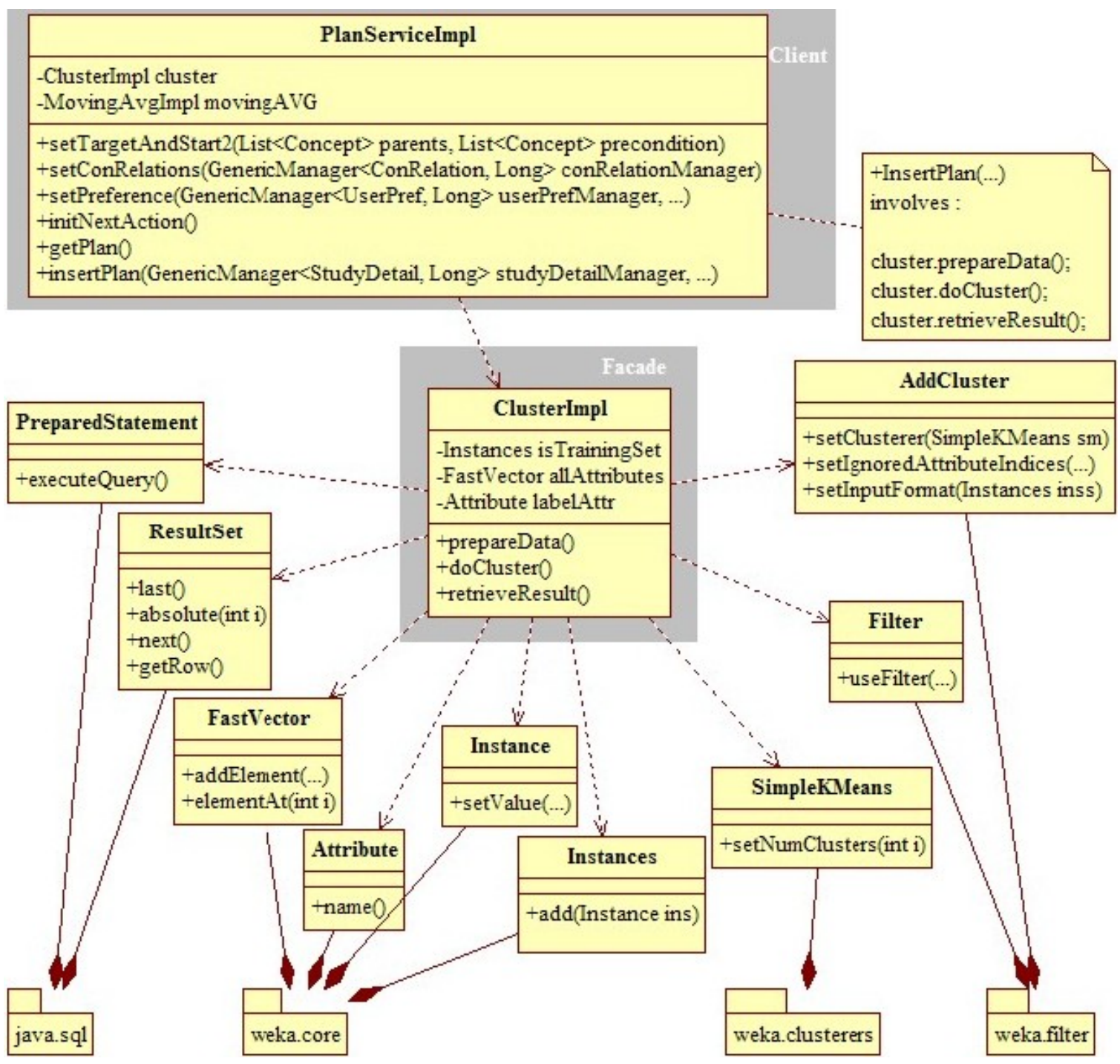

Figure 3.6: A facade pattern for encapsulating complex business logic

\subsection{Implementation}

This section introduces algorithms for learning sequences, a mechanism for estimated time, and essential functions of three functional components. 


\subsubsection{Algorithms for Learning Sequences}

One major function of ORS is the Study plan which is the core of the adaptation as described in Section 3.3.2. The learning sequence of a study plan is the result of adaptation to preference and performance. The generation of learning sequences varies when dealing with different types of preference. There are two types of preference, namely, preference without relevance and preference with relevance.

\section{Preference without Relevance}

When the preference doesn't contain the character of relevance, the Study plan uses Algorithm 1 to find a learning sequence for a particular learner initially. A Gap contains concepts which are in a learner's goal and not in the experience. The

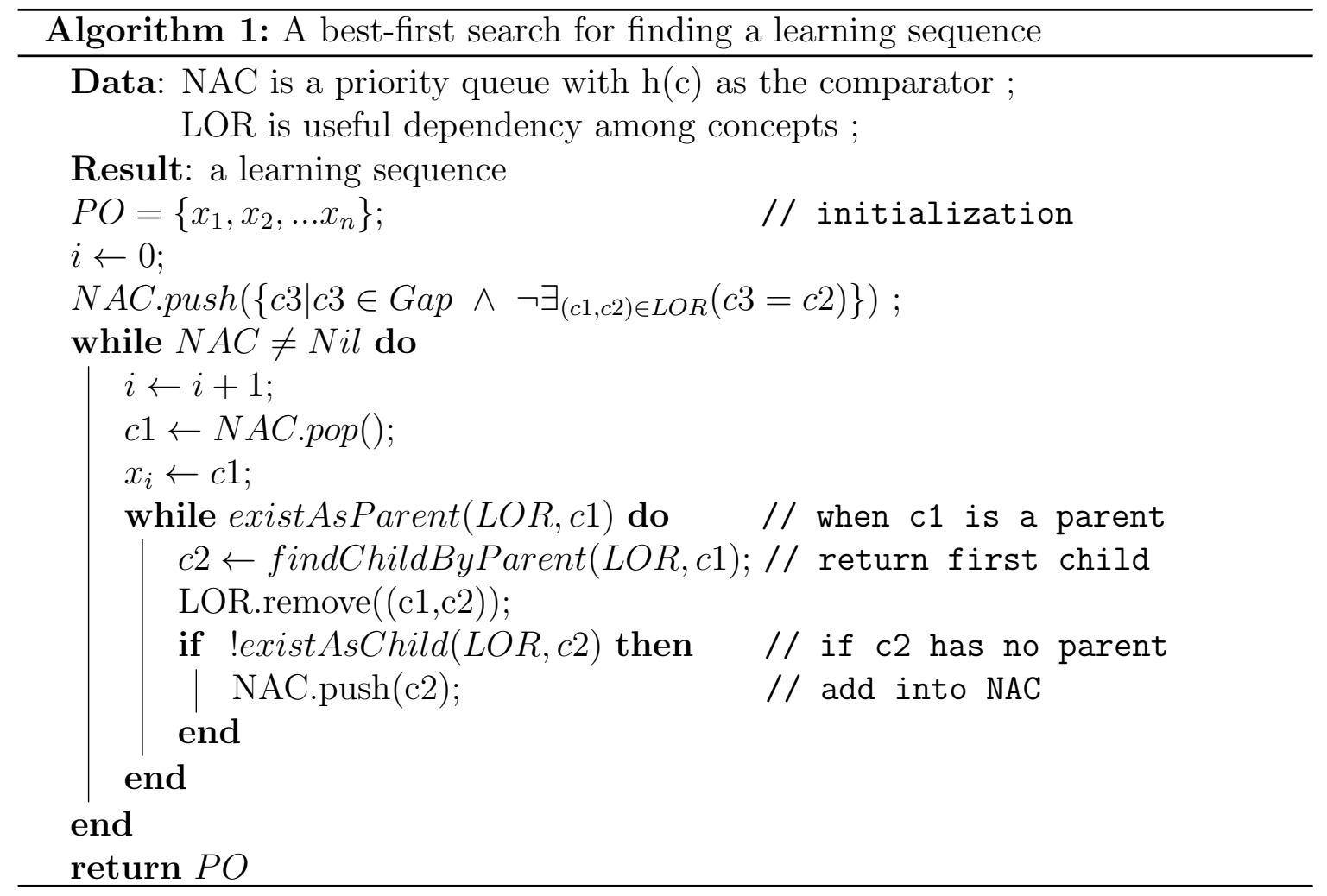


algorithm assigns all elements of a Gap to a learning sequence $\left\{x_{1}, x_{2}, \ldots x_{n}\right\}$ regarding dependency and preference. Firstly, the algorithm finds a concept with the highest priority among those concepts which belong to the Gap and whose prerequisites do not belong to the Gap. Priorities of concepts are determined by the function $h(c)$ based on preference. Subsequently, that concept is assigned to the sequence and is removed from the Gap. The dependent relationships which treat that concept as a prerequisite are also removed from the dependency. Moreover, the algorithm repeats the finding, assigning, and removing steps based on the updated Gap and dependency until the Gap is empty. Finally, each element of the learning sequence has been assigned a unique concept. The definitions of Gap and dependency (i.e., LOR) are presented in Section 3.2.3. This algorithm mainly depends on the evaluation function $h(c)$ and the priority queue $N A C$.

The evaluation function $h(c)$ determines priorities of concepts in a Gap based on preference as shown in Algorithm 2. It is a mapping Pref, PrefDetail $\rightarrow$ Integer for a concept $C \in$ Gap. We utilize a decimal integer to express the Integer, given $1<\mid$ PrefDetail $\mid<10$. The fractile reflects the priority of Pref from left to right.

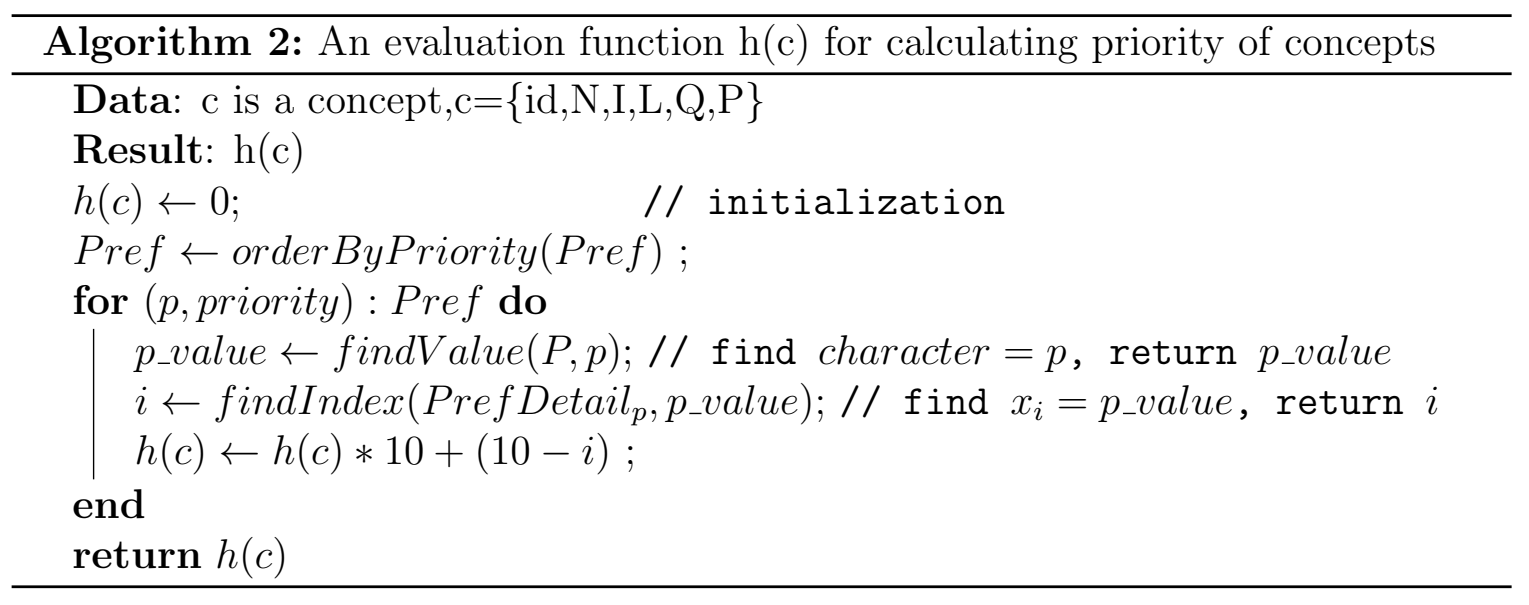


The value in each fractile expresses the priority of PrefDetail in its Pref from big to small.

The priority queue $N A C$ is used to ease the manipulation on a Gap. It contains available concepts to be chosen in the finding step of Algorithm 1. These concepts belong to the Gap and their prerequisites do not belong to the Gap. Since the NAC is a queue and has the $h(c)$ as the comparator, the top element in the queue always has the highest priority among its elements. In the assigning step, the top element is removed from the $N A C$. This leads to the result that the dependency is updated and some concepts in the Gap may be moved to the $N A C$ accordingly.

If a student achieves poor performance in learning a concept by taking its quizzes, for example, the Gap will be updated to prerequisites of this concept. A new learning sequence will be generated by invoking the algorithm again. The study plan will be updated based on the new learning sequence.

\section{Preference Containing Relevance}

When the preference contains the character of relevance, the Study plan uses Algorithm 3 to find a learning sequence for a particular learner initially. The algorithm utilizes a heuristic depth-first search method to assigns all elements of a Gap to a learning sequence $\left\{x_{1}, x_{2}, \ldots x_{n}\right\}$ regarding dependency and the learner's preference. The definitions of Gap and dependency (i.e., $L O R$ ) are presented in Section 3.2.3.

The algorithm treats a network of concepts (i.e., a Gap with its dependency) as a forest including trees. Leaves of trees are concepts which have not parent concepts. Concepts that are not parents of any one are roots of trees. The algorithm searches the forest from roots to leaves in a depth-first way. When a leaf is found, it is 


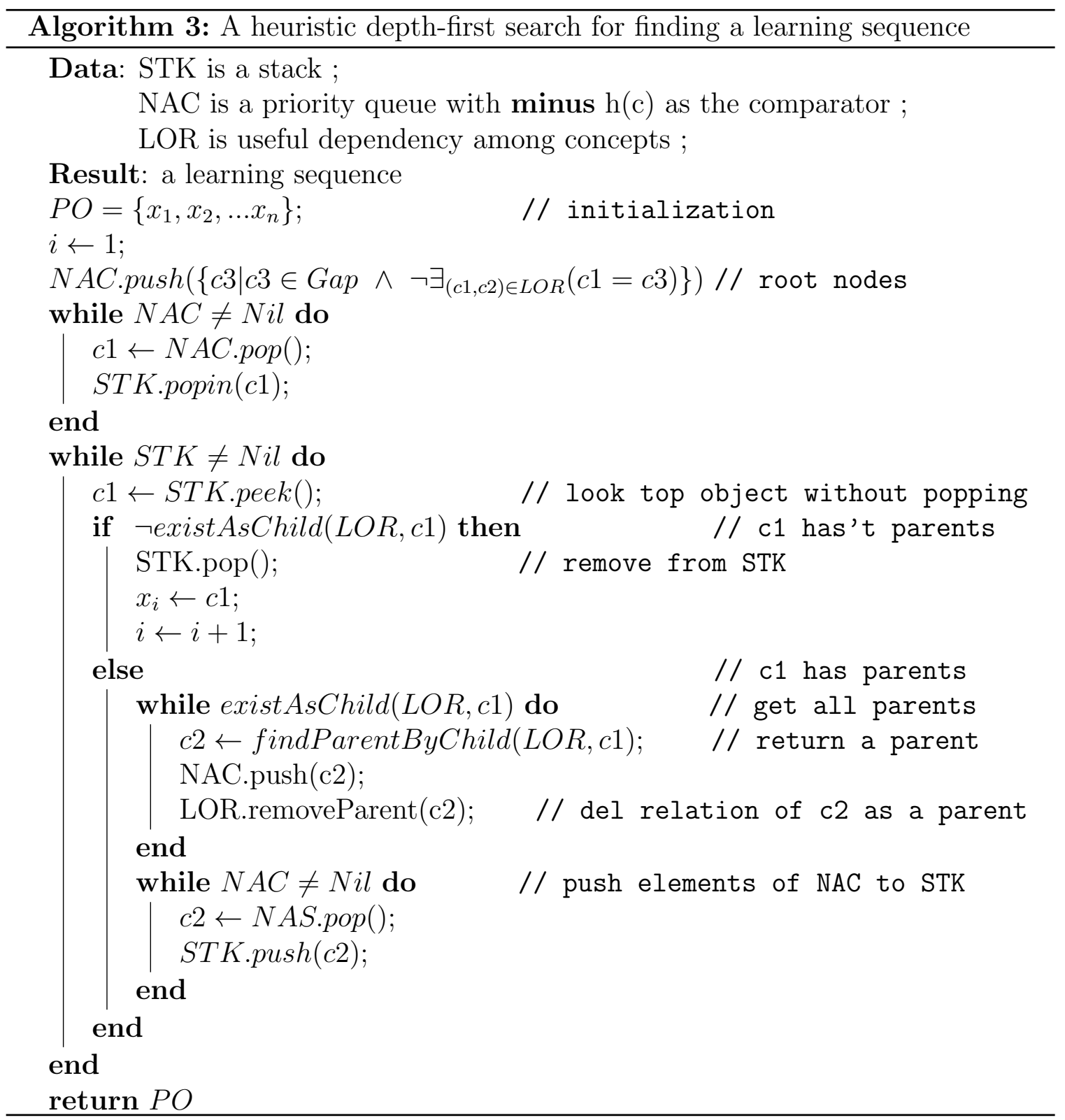

assigned to the sequence. After the assignment, the leaf is pruned from the tree, and its connections to other nodes are deleted. Let roots be the lowest nodes and leaves be the upmost nodes. Priority among roots and priority among upper nodes of a lower node are determined by the $h(c)$. The evaluation function $h(c)$ calculates the priority value of each node according to preference excluding the character of relevance. 


\subsubsection{A Mechanism for Estimated Time}

Another major function of ORS is the Estimated time described in Section 3.3.2. It estimates the future learning time for each learner to improve learning efficiency. Figure 3.7 shows that a mechanism is used to calculate estimated time of a study plan on concept by concept basis.

For the previous learners who have finished learning tasks, their time spent on each concept is recorded as well as their personality (e.g., age, education level, gender). For the learner $A$ who starts a learning task, the process of Cluster analysis identifies the actual learning time of those previous learners having similar personality with this learner. The basic notion of this process is introduced in Section 2.4.2. In the actual time, each concept has a series of data distributed on different dates. For the sake of simplicity in programming, the averages of the latest $\mathrm{N}$ similar learners are chosen to be estimated time. For each concept, the process of Moving average calculates the average time from the most recent $\mathrm{N}$ pieces of the concept's actual time. Finally, the estimated time of the learner $A$ is the result of matching between the learning sequence and those averages.

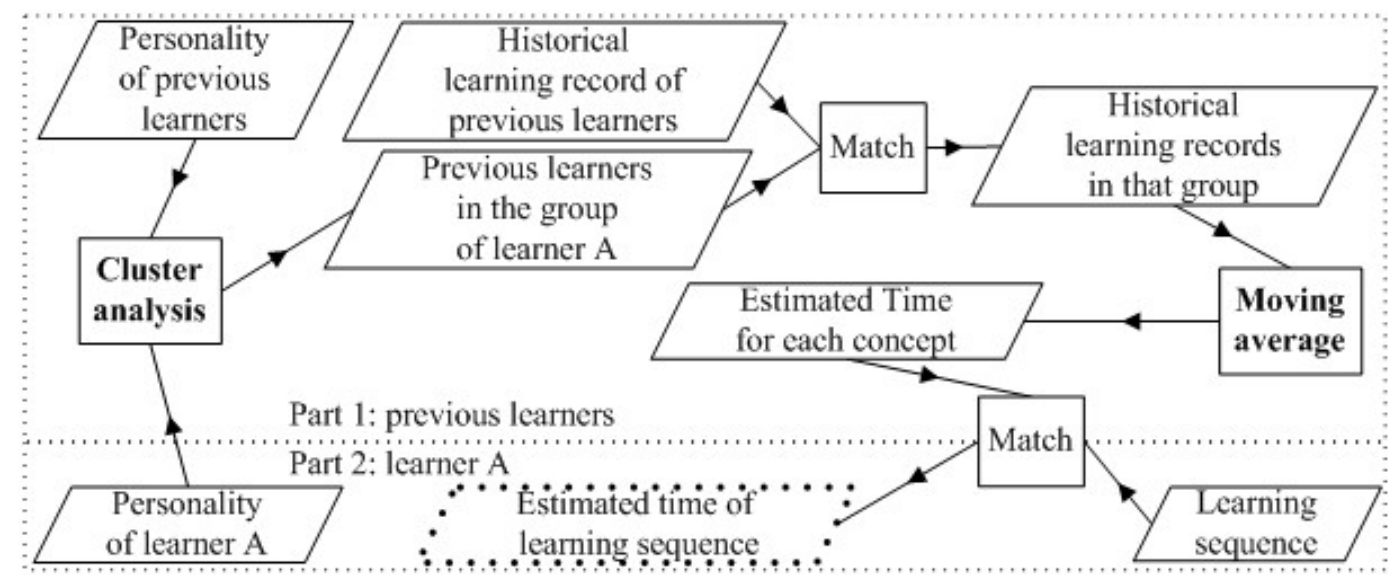

Figure 3.7: Predict the estimated time for a new learner A 
An example is presented to introduce the running of the above mechanism. Suppose learner $A$ has configured his personality, goal, experience as well as preference, and his Learning sequence contains 6 concepts. After the Cluster analysis, 20 previous learners are similar with learner A. Subsequently, their actual learning time are extracted to the Historical learning records in that group through the Match. Suppose those actual time involves 68 concepts, and each concept has a series of data distributed on different completion dates. For the sake of simplicity in programming, the Moving average calculates averages for all 68 concepts. After the Moving average, there are 68 pairs of data in the Estimated time for each concept. Each pair contains a concept and the average of its most recent 5 actual time according to completion dates. The process of Match picks up 6 pairs from the Estimated time for each concept according to concepts in the Learning sequence of learner A. The 6 pairs are stored in the Estimated time of learning sequence and used to generate a study plan for learner $A$ later.

\subsubsection{Programming Languages and Software Environment}

Java and Javascript are the two programming languages implementing ORS. In particular, two Java-based open source systems, appfuse and weka, are used. Appfuse offers the project skeleton which can be used to add new functions by programming quickly [55]. For a classic CRUD function, appfuse is able to easily generate a series of available scripts (e.g., JSPs, a Controller class, parts of XML configuration) from a POJO class, and then programmers can modify these scripts to realize desired details. It can also generate a POJO class from a database table. Some inherent functions of appfuse include authentication, authorization, user management, E-mail, 
Table 3.3: Open source softwares used in ORS

\begin{tabular}{|c|c|c|c|}
\hline Name & Version & Description & $\overline{\text { URL }}$ \\
\hline Appfuse & 2.2 .1 & $\begin{array}{l}\text { Java-based information sys- } \\
\text { tem }\end{array}$ & http://appfuse.org \\
\hline Struts & 2.3 .7 & $\begin{array}{l}\text { Web application framework } \\
\text { focusing on MVC }\end{array}$ & http://struts.apache.org \\
\hline Hibernate & 4.1 .8 & Data persistence framwork & http://www.hibernate.org \\
\hline Spring & 3.1 .3 & $\begin{array}{l}\text { Web application framework } \\
\text { focusing on application level }\end{array}$ & http://spring.io \\
\hline Dojo & 1.9 .1 & Javascript toolkit & http://dojotoolkit.org \\
\hline DWR & 2.0 .3 & $\begin{array}{l}\text { A Java library focusing on } \\
\text { AJAX }\end{array}$ & http://directwebremoting.org \\
\hline Weka & 3.6 .6 & Data mining toolkit & http://www.cs.waikato.ac.nz/ml/weka \\
\hline
\end{tabular}

and file upload. Weka provides the APIs of its clustering algorithms to support the Cluster analysis, and is used in data pre-processing, clustering, etc [31]. The system is deployed in Tomcat and the database used is MySQL.

Table 3.3 shows the open source softwares used in ORS. Struts, hibernate, spring, dojo, and DWR are also involved in the development. Appfuse has already combined with struts, hibernate, and spring. It provides the infrastructure to realize both the technical architecture and the functional architecture of ORS. Additionally, we integrate weka, dojo, and DWR into appfuse to realize several functions, such as the Record time introduced in Section 3.3.2.

\subsubsection{Implementation of the Expert Component}

This section presents some important graphical interfaces of the Expert area component, such as the Character, the Concept, the Dependency, and the Quiz. The functional requirement of each interface is described in Section 3.3.2. 


\section{Interface of the Character}

Characters are used to define soft constraints. Experts can define many characters, and each character has a set of values. Some of these values will be assigned to concepts by experts. A concept contains only one value on each character. Figure 3.8 shows typical characters of concepts. Experts can disable and enable a specific character. Figure 3.9 shows details of a character (e.g., the difficulty). Experts can CRUD its details if necessary.

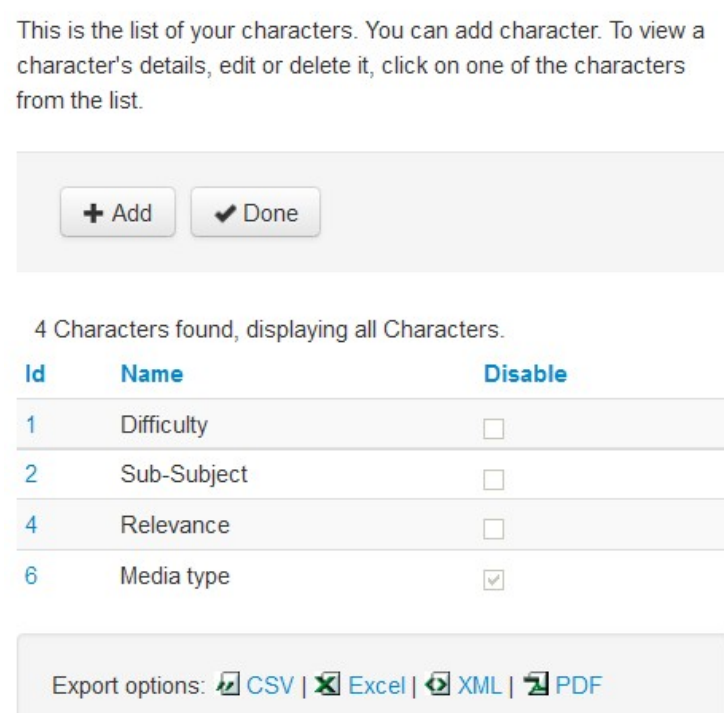

Figure 3.8: View a list of characters

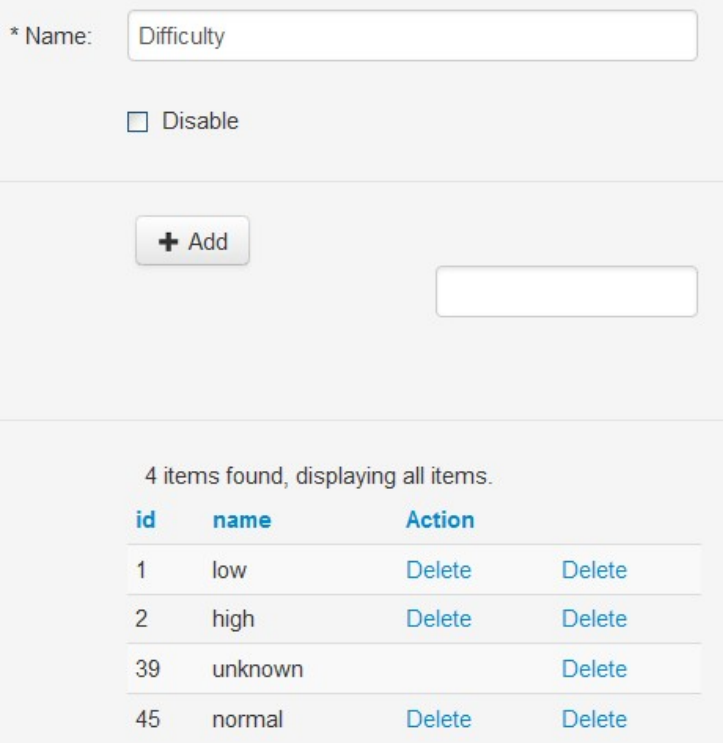

Figure 3.9: Edit details of a character

\section{Interface of the Concept}

Experts can CRUD a concept in ORS. As shown in Figure 3.10, the Concept interface presents three parts of information, namely, basic info, characters, and quizzes. Basic info contains the name of a concept, description, and links of related papers or content. Characters identify the detail of each character on that concept. Quizzes 


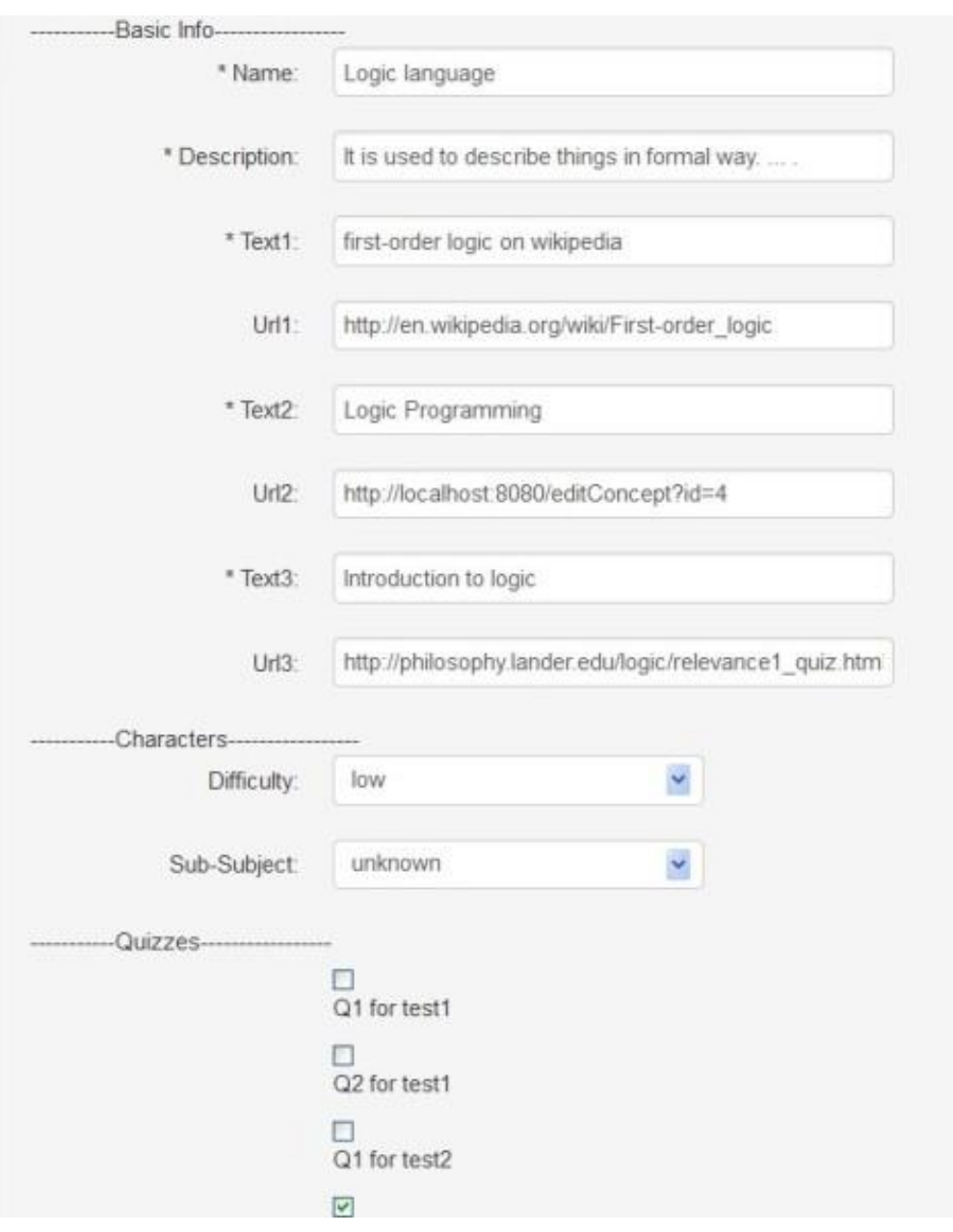

Figure 3.10: Edit a concept

connect specific questions with that concept. A concept can be assigned values of several characters. It has one and only one value on each character.

\section{Interface of the Dependency}

An item of dependency is also described as a learning order relationship or a prerequisite. Experts can CRUD an item of dependency. Dependency connects the prerequisite concepts for each concept if such relationships exist. An item of dependency consists of two parts, namely, a parent concept and a child concept. Students 


\begin{tabular}{|c|c|c|c|c|}
\hline \multicolumn{4}{|c|}{103 Dependencies found, displaying 1 to 25 . } & \multirow{2}{*}{$\begin{array}{l}\text { [First/Prev] 1, 2, 3, 4, } 5 \text { [Next/Last] } \\
\text { ConceptName }\end{array}$} \\
\hline Id & ParentConld & ParentConName & Conceptid & \\
\hline 16 & 1 & Concept & 6 & Concept in an information table \\
\hline 9 & 2 & Information table & 3 & Equivalence Relation in Information table \\
\hline 12 & 2 & Information table & 4 & Logic language \\
\hline 14 & 2 & Information table & 5 & Means of formula \\
\hline 17 & 2 & Information table & 6 & Concept in an information table \\
\hline
\end{tabular}

Figure 3.11: View a list of dependency

are required to master the parent concepts before they learn a child concept. Figure 3.11 shows a list of dependency among rough sets concepts in ORS.

After an expert trying to change an item of dependency, the ORS checks whether or not there is an infinite loop in dependency caused by the change. If not, that change will be stored in the system; Otherwise, the change will be canceled.

\section{Interface of the Quiz}

Several quizzes always accompany a concept. Experts create multiple quizzes. A quiz consists of two parts, namely, the question and the answers. The answers include not only the right solutions, but also the wrong ones. Figure 3.12 shows the interface of editing a quiz. For a quiz belonging to choice selection. The question and answers are pictures containing text.

\subsubsection{Implementation of the Tutor Component}

This section presents some important graphical interfaces of the Tutor area component, such as the Range, the Home page, the Record time, and the Estimated time. The functional requirement of each interface is described in Section 3.3.2. 


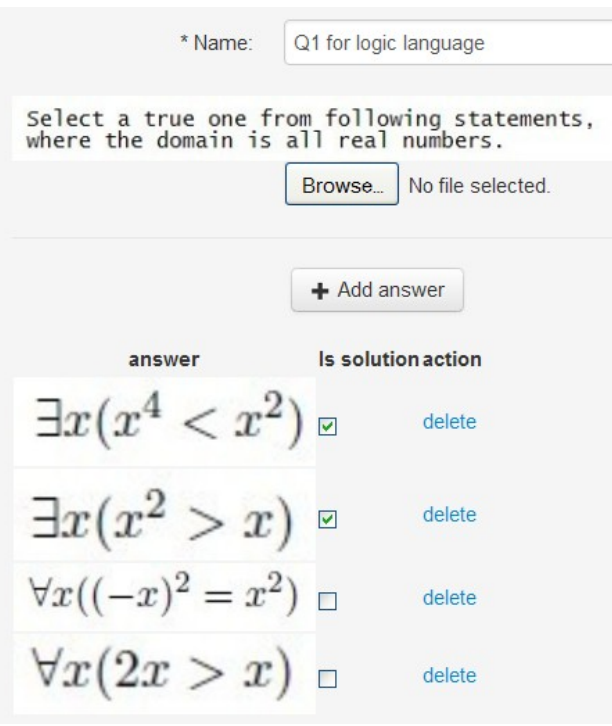

Figure 3.12: Edit a quiz

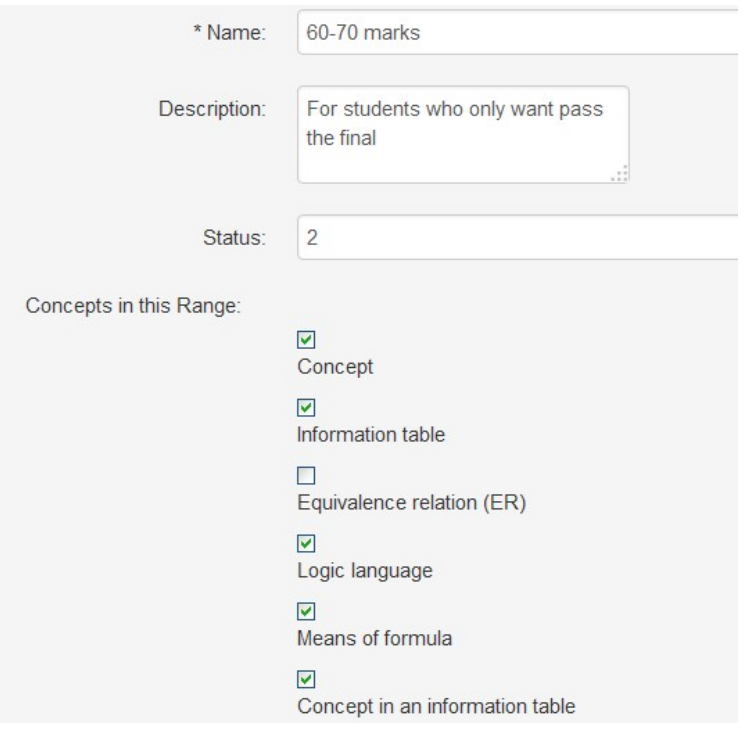

Figure 3.13: Define a range from concepts

\section{Interface of the Range}

A range points to a set of concepts. Tutors define a series of ranges. With these ranges, it is easier for students to select their learning objectives. Typical ranges in ORS include 60-70 marks, 70-80 marks, 80-100 marks, and Pursuing excellence. Figure 3.13 shows the interface of editing a range. Tutor can change the concepts of a range by selecting and canceling them in the concept list.

\section{Interface of the Record Time}

The Record time is implemented in the Working place of the student area. It provides three ways to record the actual learning time spent on a concept: (1) students press the Pause button in the Working place; (2) students press the Done button in the Working place; and (3) the ORS automatically records if students ignore the periodical messages.

The system prompts a message to a learner every thirty seconds as shown in 


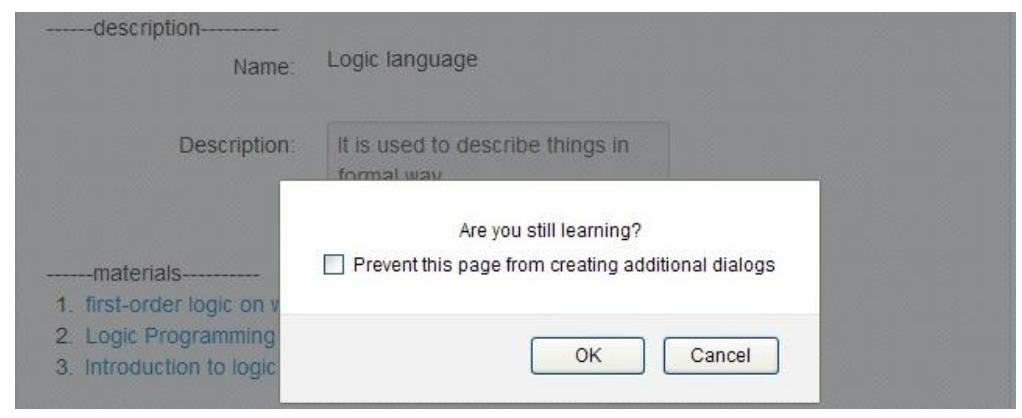

Figure 3.14: Popup message for recording actual study time of students

Figure 3.14. If no action happens, the system supposes that the learner is absent. The time after the notification will not be added. If pressing the $O K$, the system starts to record time again. If pressing the Cancel, the system goes to the home page.

\section{Interface of the Estimated Time}

The Estimated time is a function running in the back scene. It calculates the estimated time for every student by using the mechanism presented in Figure 3.7. The result is used in the Study plan to generate study plans for students.

\section{Interface of the Home Page}

This is the home page for tutors, as well as experts and students. Figure 3.15 shows the interface. Users can click related links to access different functions.

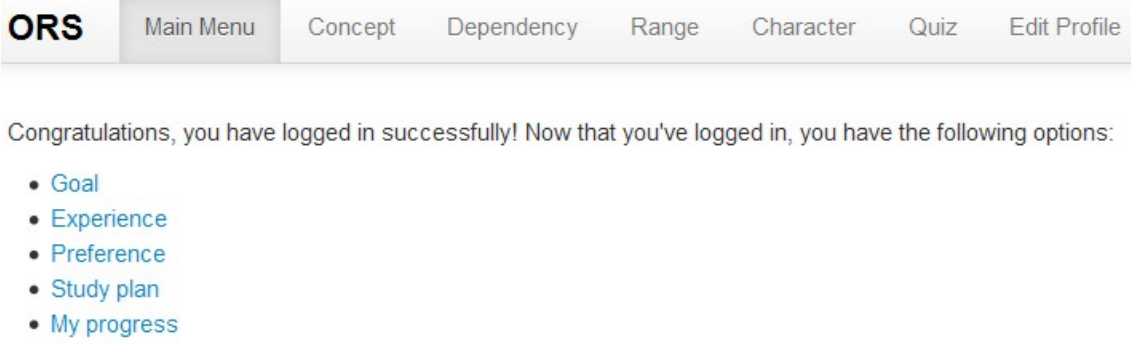

Congratulations, you have logged in successfully! Now that you've logged in, you have the following options:

- Goal

- Experience

- Preference

- Study plan

- My progress

Figure 3.15: Home page of ORS 


\subsubsection{Implementation of the Student Component}

This section presents some important graphical interfaces of the Student area component, such as the Goal, the Experience, the Preference, the Study plan, the Working place, and the My progress. The functional requirement of each interface is described in Section 3.3.2.

\section{Interface of the Goal}

A goal contains learning objectives of a student, and includes a set of concepts. There are two ways for a student to define a goal. One way is the student chooses a range from the range list. Another way is the student chooses several concepts directly from the concept list. In this way, the student only has to choose the concepts roughly, and dependent concepts of selected ones will be chosen automatically. When the student chooses Concept in an information table, for example, the related seven

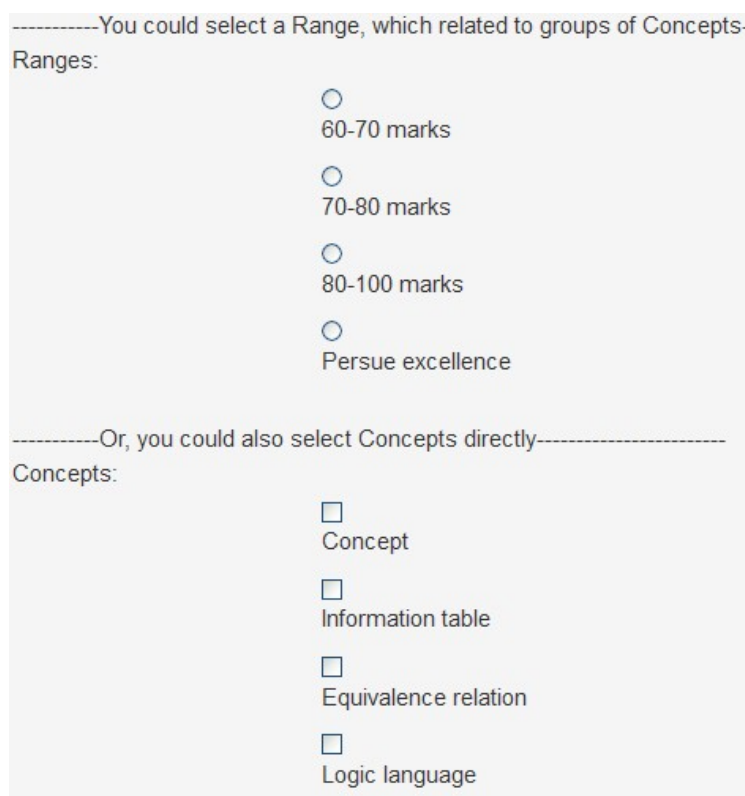

Figure 3.16: Set a goal from two ways

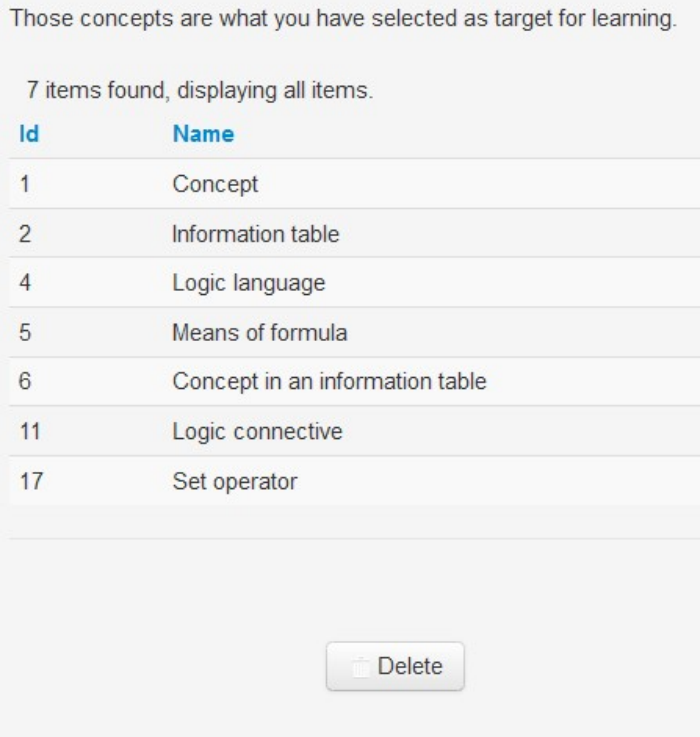

Figure 3.17: Concepts of a goal 
concepts are automatically selected based on dependency.

Figure 3.16 shows the interface of defining a goal. Students can select their goals from ranges or the concept list. Figure 3.17 shows the result after selection in the above example .

\section{Interface of the Experience}

An item of experience is a set of concepts which have already been mastered by the student after defining a goal. Suppose the student has known Logic connective in the above example.

Figure 3.18 shows the interface of marking concepts as experience. In the example, there are seven concepts in the goal. However, there are only three concepts without prerequisites. The student can mark his experience from the three concepts as shown in Figure 3.18. We assume that the student selects Logic connective and clicks the Next. The result is shown in Figure 3.19.

After marking the concepts as experience, some items of the dependency may be

Please select the concepts you have known.

Concepts:
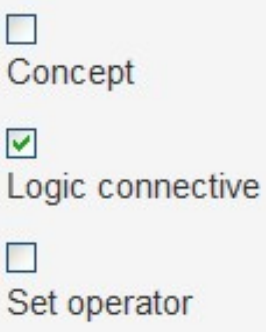

Next

Figure 3.18: Mark concepts as experience
7 items found, displaying all items.

\begin{tabular}{|ll|}
\hline Id & Name \\
\hline 1 & Concept \\
\hline 2 & Information table \\
\hline 4 & Logic language \\
\hline 5 & Means of formula \\
\hline 6 & Concept in an information table \\
\hline 11 & Logic connective (known) \\
\hline 17 & Set operator \\
\hline
\end{tabular}

Figure 3.19: A goal with experience 
ignored. This leads to the result that other concepts may be available to be marked. The student can mark experience from bottom to top in this way.

\section{Interface of the Preference}

Students define preference to personalize their learning sequences. An item of preference has prioritized constraints based on character values on concepts. It consists of two hierarchies, namely, priority of different characters and priority of character values in each character.

Figure 3.20 shows the interface of how to define priority among different characters. Initially, a student can choose 0 or $\mathrm{N}$ characters. Subsequently, the student can define the priority by dragging characters up and down. In the example, the Difficulty character has higher priority than the Sub-subject.

Figure 3.21 shows the interface of how to define priority inside a character. A student can define the priority by dragging values of that character up and down. For example, we click the modify button of the difficulty character in Figure 3.20. A popup window is highlighted and shows the character's values in Figure 3.21. These values can be dragged. In this case, the low has the highest priority, while the

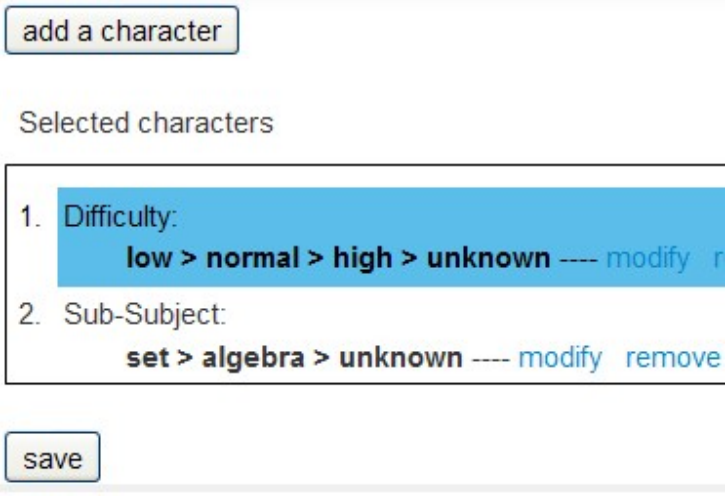

Figure 3.20: Priority among characters

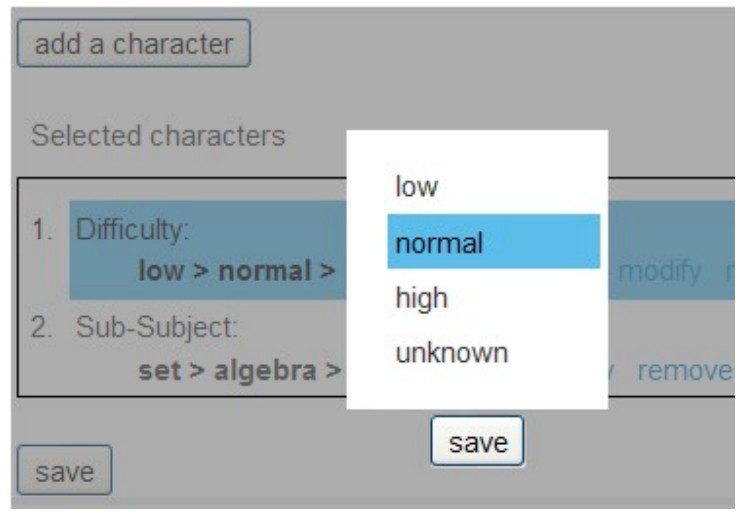

Figure 3.21: Priority of character values 
unknown is the lowest; the normal has higher priority than the high.

\section{Interface of the Study Plan}

Study plans are the main output of the learning model. It is used to guide learners to study rough sets effectively and efficiently. As shown in Figure 3.22, a study plan mainly consists of two parts, namely, the learning sequence and the estimated time.

\begin{tabular}{llllll} 
Learning Sequence & Concept & Created Date & Updated Date & Estimated Time Status \\
001 & Set operator & $10-06-2014$ & $10-06-2014$ & 12 & 0 \\
\hline 002 & Information table & $10-06-2014$ & $10-06-2014$ & 18 & 0 \\
\hline 003 & Logic language & $10-06-2014$ & $10-06-2014$ & 27 & 0 \\
\hline 004 & Means of formula & $10-06-2014$ & $10-06-2014$ & 12 & 0 \\
\hline 005 & Concept & $10-06-2014$ & $10-06-2014$ & 16 & 0 \\
006 & Concept in an information table & $10-06-2014$ & $10-06-2014$ & 9 & 0 \\
\hline Total Estimated Time: & & & & 94.0 &
\end{tabular}

Figure 3.22: A study plan containing the learning sequence and estimate time

The learning sequence guides students to learn concepts one by one rationally. It has considered dependency among concepts, a student's goal, experience, and preference. The learning sequence is updated if the student achieves poor performance on a quiz. The estimated time tells the learner how long he/she probably requires to study each concept. It helps students schedule learning paces and improve learning efficiency.

\section{Interface of the Working Place}

Students learn concepts and take quizzes in the Working place. Figure 3.23 shows the interface of how a student learns a concept. The student can study description of the concept and links of content. In the learning period, the student can pause the study by pressing the Pause if required. The student can continue to study that concept when available in the future. 


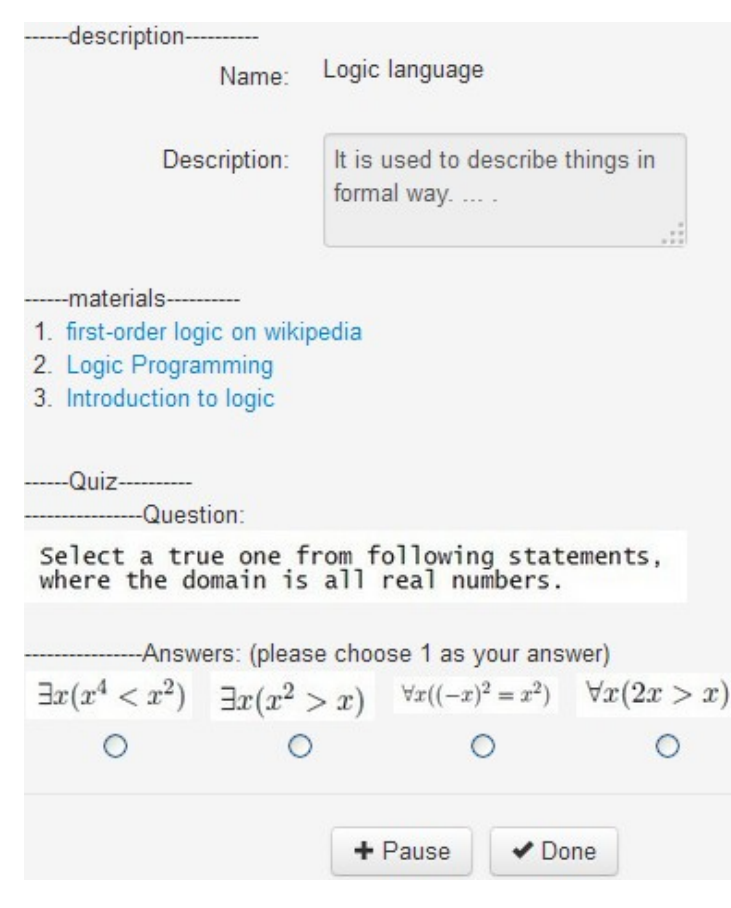

Figure 3.23: Learn and test a concept

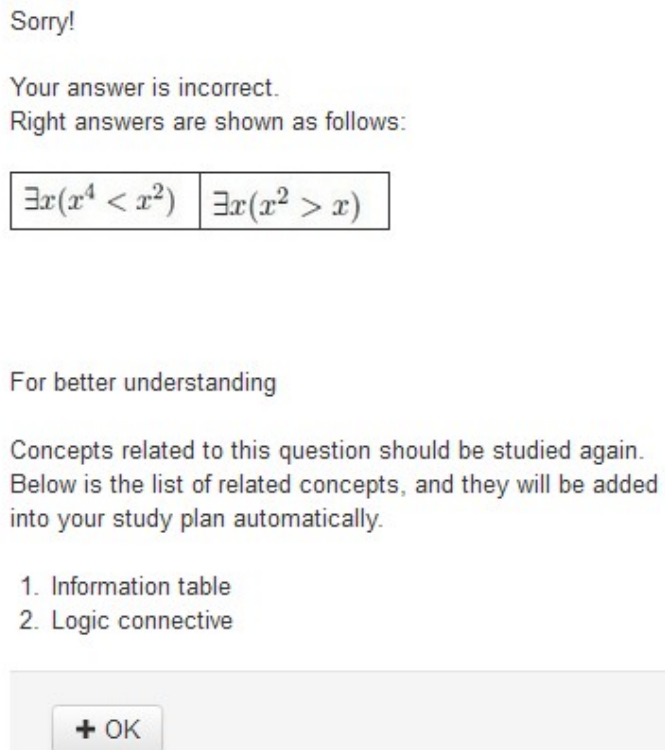

Figure 3.24: Notification of a failed quiz

After learning a concept properly, the student should take its quizzes by reading their questions and selecting right answers. The student is notified if wrong answers are made in a quiz. Figure 3.24 shows the details of notification. This notification provides not only the quiz's right answers, but also the prerequisites of the concept related to this quiz.

\section{Interface of the My Progress}

The progress is generated after a student starting to learn concepts following his/her study plan. It reflects the learner's current learning status. As shown in Figure 4.2, the progress mainly consists of two parts, namely, the finished part and the unfinished part.

The finished part presents concepts which have been mastered by a student. The time spent on learning these concepts is also displayed. The unfinished part shows 
Have finished:

This is your current progress. Please continue to finish your job. Or, you could delete it.

\begin{tabular}{llllll} 
Learning Sequence & Concept & Created Date & Updated Date & Time Spent & Status \\
\hline 001 & Set operator & $19-02-2014$ & $19-02-2014$ & 12 & 2 \\
\hline 002 & Information table & $19-02-2014$ & $19-02-2014$ & 18 & 2
\end{tabular}

Need to learn:

\begin{tabular}{llllll} 
Learning Sequence & Concept & Created Date & Updated Date & Estimated Time Status \\
\hline 003 & Logic language & $19-02-2014$ & $19-02-2014$ & 27 & 1 \\
\hline 004 & Means of formula & $19-02-2014$ & $19-02-2014$ & 12 & 0 \\
\hline 005 & Concept & $19-02-2014$ & $19-02-2014$ & 16 & 0 \\
\hline 006 & Concept in an information table & $19-02-2014$ & $19-02-2014$ & 9 & 0 \\
Total Estimated Time: & & & 64.0 &
\end{tabular}

Figure 3.25: Progress showing the finished part and unfinished part to a student

how many remaining concepts the student needs to learn in the future. The estimated time is offered to help the student schedule his/her learning paces. 


\section{Chapter 4}

\section{Demonstrative Examples}

This chapter demonstrates how a student gets an adapted study plan based on the student's preference and performance. Firstly, we use a simple example to show adaptation in the whole learning process. Secondly, a complex example illustrates how the learning sequence adapts to preference containing multiple characters. Subsequently, a comparison between ORS and some existing Web-based learning systems is presented.

\subsection{Adaptation in the Whole Learning Process}

In this section, we illustrate when and how adaptation to preference and performance is made. The learning objective is only one concept. The preference chosen contains only one character.

\subsubsection{Planning with Preference and Estimated Time}

Suppose a student wants to learn a specific concept, called Concept in an information table. When the student chooses Concept in an information table in ORS, 


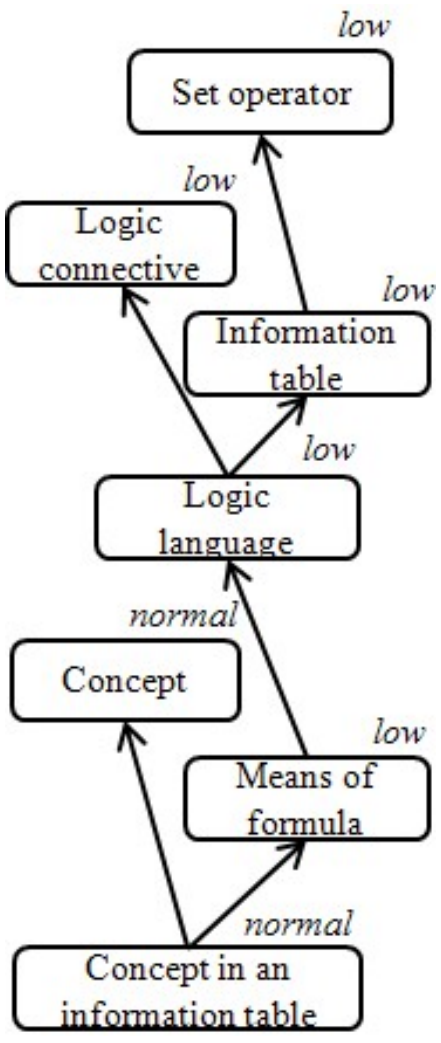

(a) Goal before experience

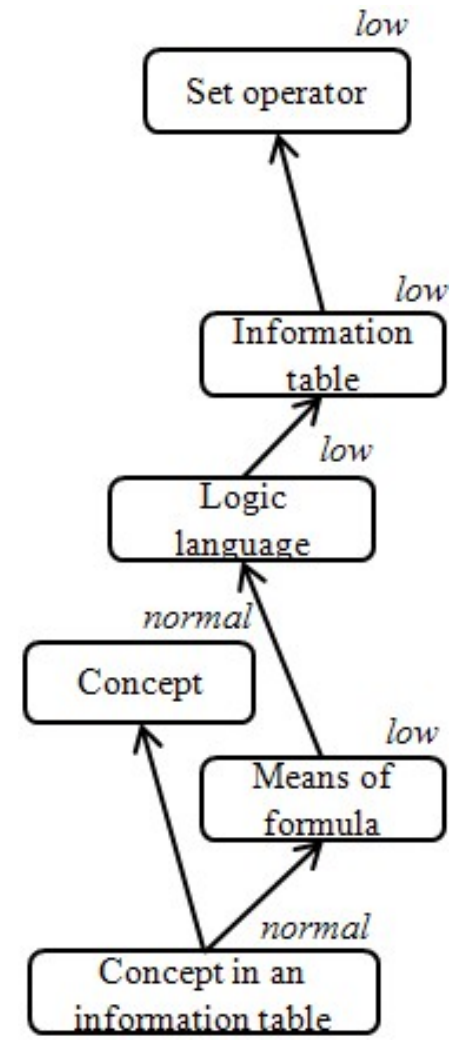

(b) Goal after experience

\section{Before preference:}

more than 2 possible leaming sequences

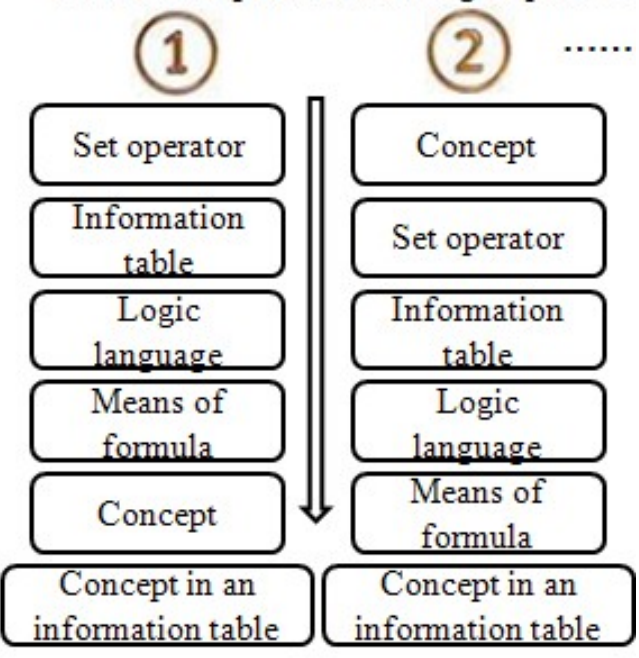

After preference (low>nomal >high):

(1) is the only learning sequence

(c) Adaptation to preference

Figure 4.1: Effect of experience and preference on a goal

the related 7 concepts are automatically selected based on the dependency. These concepts, the dependency, and their difficulty levels on the difficulty character are shown in Figures 4.1 (a). We assume that the student has known Logic connective and marks it as the experience. What the student needs to learn are the 6 concepts presented in Figures 4.1 (b).

Adaptation to preference is highlighted in Figures 4.1 (c). Before applying the preference, there are multiple available learning sequences. After the student selecting and configuring the character of difficulty as preference (e.g., from low to high), there is only one learning sequence left.

A study plan is generated by ORS for the student as shown in Table 4.1. The 
Table 4.1: A study plan including a learning sequence and estimated time

\begin{tabular}{|c|l|c|}
\hline Learning Sequence & \multicolumn{1}{|c|}{ Concept } & $\begin{array}{c}\text { Estimated Time } \\
\text { (minutes) }\end{array}$ \\
\hline 001 & Set operator & 12 \\
\hline 002 & Information table & 18 \\
\hline 003 & Logic language & 27 \\
\hline 004 & Means of formula & 12 \\
\hline 005 & Concept & 16 \\
\hline 006 & Concept in an information table & 9 \\
\hline Total: & & 94 \\
\hline
\end{tabular}

learning sequence and the estimated time are presented in different columns. The learning sequence helps the student learn rationally. The estimated time is helpful to schedule the student's learning paces and improve learning efficiency, since it can help the student decrease interruption caused by time shortage in learning a concept.

\subsubsection{Learning Sequences Adapted to Performance}

Based on the above study plan, we assume that the student has learned the concepts of Set operator and Information table successfully. The progress is shown in Figure 4.2. The finished part lists the above 2 concepts, while the unfinished part

Have finished:

\begin{tabular}{l} 
Learning Sequence \\
001 \\
\hline 002
\end{tabular}

Need to learn:

\begin{tabular}{|ll}
\hline Learning Sequence & Concept \\
\hline 003 & Logic language \\
\hline 004 & Means of formula \\
\hline 005 & Concept \\
\hline 006 & Concept in an information table \\
\hline
\end{tabular}

Total Estimated Time:
This is your current progress. Please continue to finish your job. Or, you could delete it.

\begin{tabular}{lllll} 
Concept & Created Date & Updated Date & Time Spent & Status \\
\hline Set operator & $19-02-2014$ & $19-02-2014$ & 12 & 2 \\
\hline Information table & $19-02-2014$ & $19-02-2014$ & 18 & 2
\end{tabular}

Figure 4.2: Learning progress reflecting how many concepts are mastered and left 


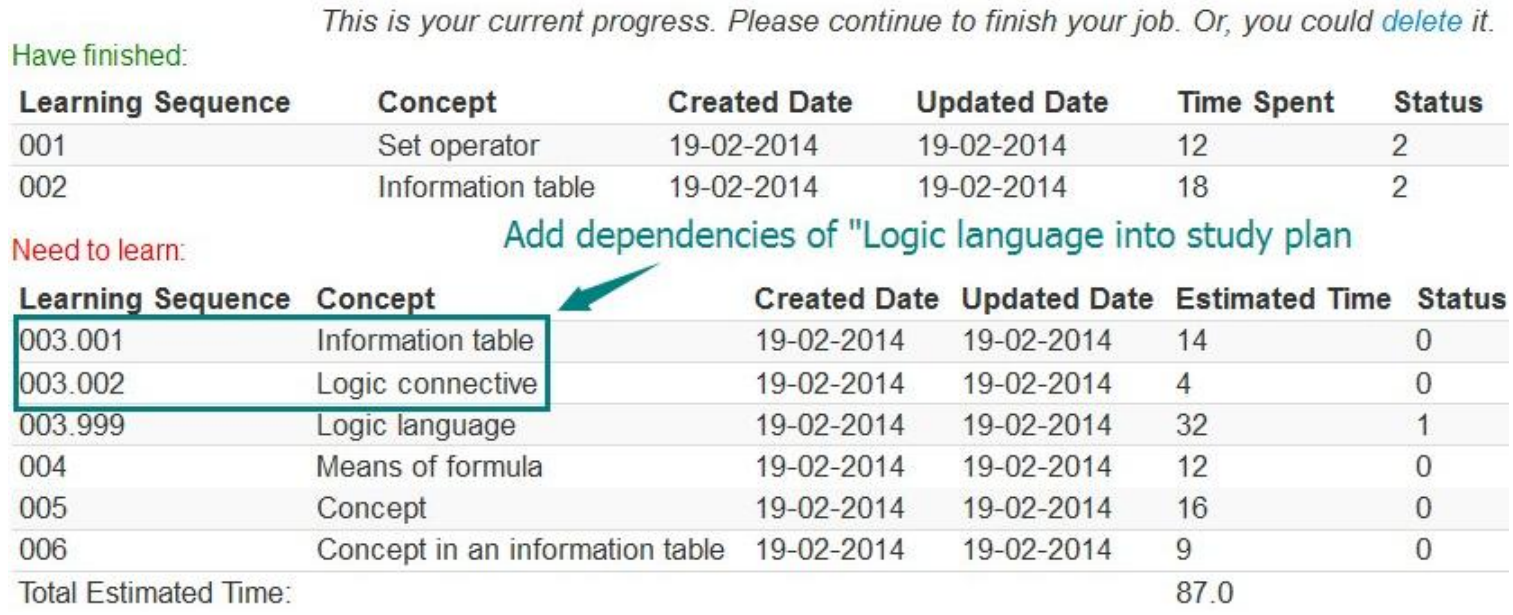

Figure 4.3: Adaptation to performance leading to the increase of unfinished part

contains the left 4 concepts. Subsequently, the student starts to learn Logic language but fails to pass its quizzes.

In this case, adaptation to performance is made due to the failure. The ORS notifies and directs the student to learn the prerequisite concepts of Logic language again, namely, Information table and Logic connective. These two concepts are added into the study plan as shown in Figure 4.3. By observing the difference of values in the first column, the student knows which concepts have been added.

From this example, the student can learn rough sets rationally and efficiently with the adapted study plan, which is updated according to preference and performance in the whole learning process.

\subsection{Adaptation to Preference Containing Multiple}

\section{Characters}

In this section, we illustrate adaptation to preference having multiple characters. The learning objectives are two irrelevant concepts. The preference chosen contains 
two characters.

\subsubsection{Defining Multiple Characters as Preference}

Suppose a student wants to learn two irrelevant concepts, namely, DTRS and Reduct using DM (Discernibility matrix). When the student chooses the two concepts in ORS, the 24 concepts are automatically selected based on the dependency.

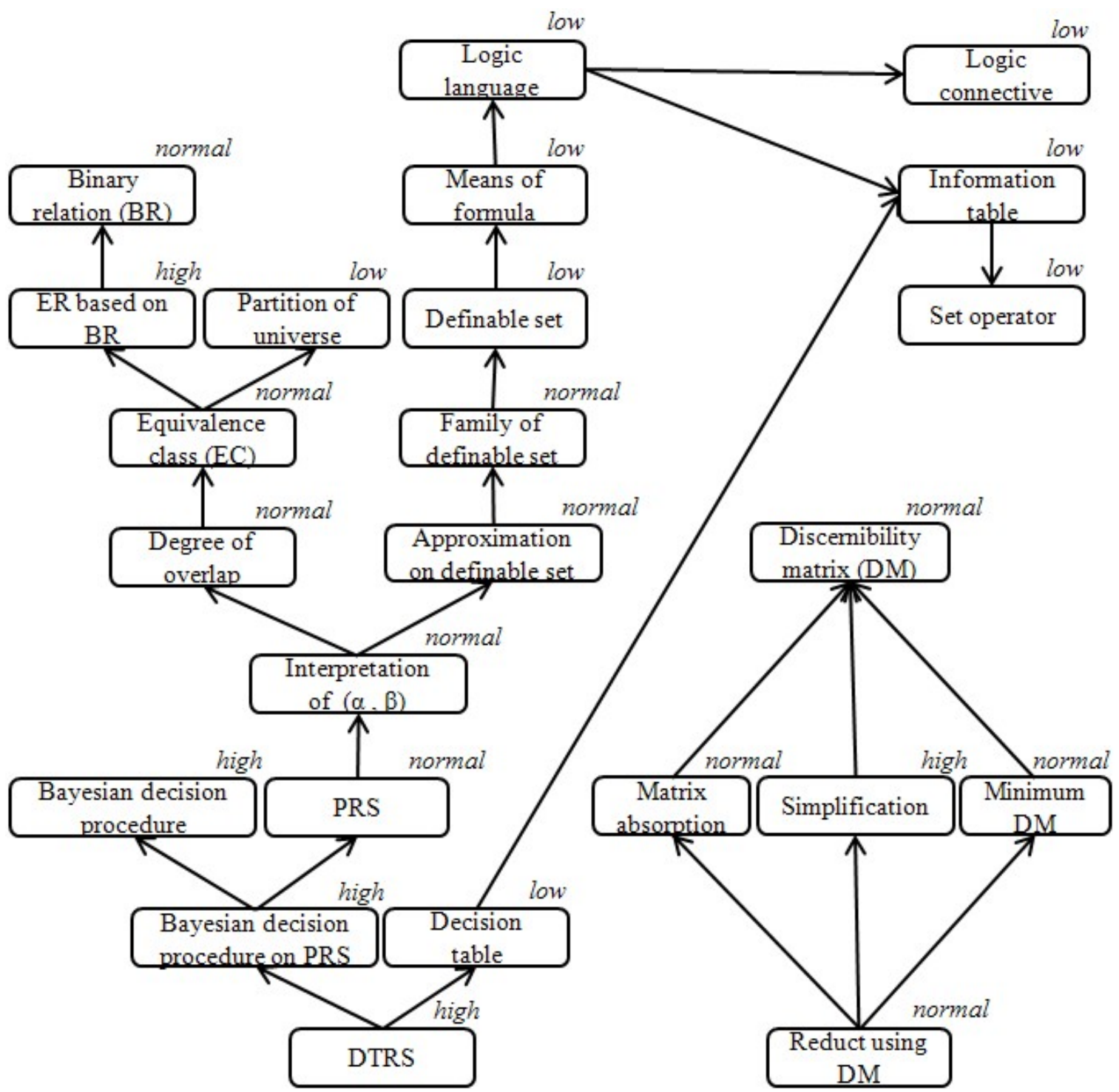

Figure 4.4: Related concepts of DTRS and Reduct using DM 


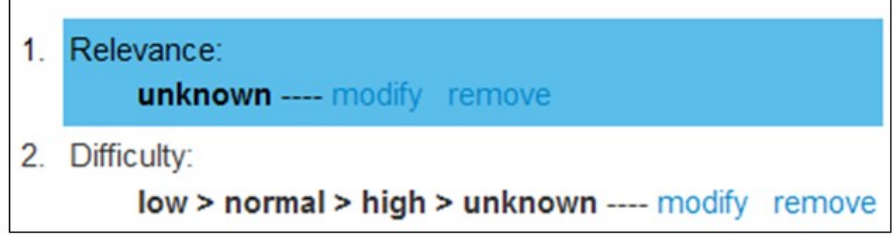

Figure 4.5: Preference containing multiple characters

These concepts, the dependency, and their difficulty levels on the difficulty character are shown in Figure 4.4. The concepts connected to Reduct using DM are isolated from the concepts related to DTRS. We also assume the student doesn't have any experience on those concepts.

We assume that the student selects the characters of relevance and difficulty as preference. As shown in Figure 4.5, the student defines that the relevance character has higher priority than the difficulty. This leads the learning sequence's generation to satisfy the relevance character firstly. After the relevance being satisfied, the difficulty character is considered as best it can.

The relevance character leads the system to sort a series of concepts by using a dept-first strategy. It only has one value (i.e., the unknown) which does not require to be configured. We treat a set of concepts with dependency as a forest including trees. Leaves of trees are concepts which have not parent concepts, such as Binary relation $(B R)$ in Figure 4.4. Concepts that are not parents of any one are roots of trees, such as Reduct using DM and DTRS in Figure 4.4. Let roots be the lowest nodes and leaves be the upmost nodes. A leaf always has higher priority than its lower node. After a leaf is assigned to the sequence, the leaf is pruned from the tree as well as its connections. The search backs to the leaf's (former) lower node, and continues to find and prune other leaves of this node. If no leaf exists, that node becomes a leaf 
Table 4.2: A study plan adapted to preference containing multiple characters

\begin{tabular}{|c|c|c|}
\hline Learning Sequence & Concept & $\begin{array}{c}\text { Estimated Time } \\
\text { (minutes) }\end{array}$ \\
\hline 001 & Discernibility matrix (DM) & 9 \\
\hline 002 & Matrix absorption & 15 \\
\hline 003 & Minimum DM & 14 \\
\hline 004 & Simplification & 11 \\
\hline 005 & Reduct using DM & 16 \\
\hline 006 & Set operator & 12 \\
\hline 007 & Information table & 18 \\
\hline 008 & Decision table & 8 \\
\hline 009 & Logic connective & 5 \\
\hline 010 & Logic language & 27 \\
\hline 011 & Means of formula & 12 \\
\hline 012 & Definable set & 10 \\
\hline 013 & Family of definable set & 10 \\
\hline 014 & Approximation on definable set & 11 \\
\hline 015 & Partition of universe & 11 \\
\hline 016 & Binary relation $(\mathrm{BR})$ & 19 \\
\hline 017 & ER based on BR & 6 \\
\hline 018 & Equivalence class (EC) & 20 \\
\hline 019 & Degree of overlap & 13 \\
\hline 020 & Interpretation of $(\alpha, \beta)$ & 18 \\
\hline 021 & PRS & 11 \\
\hline 022 & Bayesian decision procedure & 21 \\
\hline 023 & Bayesian decision procedure on PRS & 22 \\
\hline 024 & DTRS & 41 \\
\hline \multicolumn{2}{|l|}{ Total: } & 360 \\
\hline
\end{tabular}

and will be assigned and pruned.

The difficulty character leads the system to rank a series of concepts according to their difficulty levels. These levels include the low, the normal, the high, and the unknown. The student can configure an order of those levels. In this case, the student defines the order as low $>$ normal $>$ high $>$ unknown. It means concepts with the level of low have the highest priority and the unknown leads to lowest priority.

A study plan is generated by ORS for the student as shown in Table 4.2. The 
learning sequence is the result of adaptation to preference with both the relevance character and the difficulty character.

\subsubsection{Analysis of Learning Sequences}

We find related concepts are ordered together from the learning sequence in Table 4.2. For example, the concepts related to Reduct using DM are supposed to be studied firstly. Steps from 001 to 005 guide the learning of Reduct using DM. Steps from 006 to 024 guide the learning of DTRS. The separation of two group steps is the result of the relevance character. Without choosing that character, the steps of two group concepts may interlace although the dependency is satisfied. For example, ER based on BR may be the next step of Discernibility matrix (DM).

We also find the effect of the difficulty character after the relevance being satisfied. For example, the concepts of Reduct using DM are supposed to be studied priori to those of DTRS. In terms of the difficulty character, Reduct using DM has a level of normal, and has higher priority than DTRS. In another example, the concepts of PRS are supposed to be studied earlier than those of Bayesian decision procedure. Steps from 009 to 021 guide the learning of PRS. Step 022 is for the learning of Bayesian decision procedure. Both PRS and Bayesian decision procedure are prerequisite concepts of Bayesian decision procedure on PRS. Their difficulty levels make that priority.

From this example, the student can select and configure multiple characters as his/her preference. With this capability, the student is able to personalize the learning sequence in more ways. 


\subsection{Comparing ORS with Existing Systems}

The features of ORS can be highlighted by comparing with the 7 existing Webbased learning systems for computer science subjects. The comparison was performed by investigating system functions according to the definition of WLSS introduced in Section 2.3.2. The following 4 main functions of WLSS and their details were reviewed to make such comparison.

1. Complexity of learning support: Supporting complex learning actions and tools, including ontology, simulator, practice, game, multimedia, and estimated time.

2. Adaptability support : Sequencing learning materials due to learner data including goal, experience, preference, and performance.

3. Interaction support : Communication forms between learners and systems, including annotation, asking questions, feedback, and collaboration.

4. Assessment support : Methods of evaluating learner understanding, including exercise and test.

Table 4.3 shows the difference between ORS and existing Web-based learning systems. The row header indicates the 4 main functions for comparison, and the column header corresponds learning subjects of the 7 existing systems as well as

ORS. Each data cell indicates what detail functions a system has on a particular main function.

The system for artificial intelligence addresses the 4 main functions evenly. Systems for theory of computation, robotic, and information retrieval emphasize the complexity of learning support. The system for system analysis emphasizes both 
Table 4.3: Comparing ORS with existing systems for computer science subjects

\begin{tabular}{|c|c|c|c|c|}
\hline Subjects & $\begin{array}{c}\text { Complexity } \\
\text { of learning } \\
\text { support }\end{array}$ & $\begin{array}{c}\text { Adaptability } \\
\text { support }\end{array}$ & $\begin{array}{c}\text { Interaction } \\
\text { support }\end{array}$ & $\begin{array}{c}\text { Assessment } \\
\text { support }\end{array}$ \\
\hline Theory of computation [34] & Ontology & & Feedback & \\
\hline Theory of computation [21] & $\begin{array}{c}\text { Simulator } \\
\text { Practice } \\
\text { Game }\end{array}$ & Goal & $\begin{array}{c}\text { Feedback } \\
\text { Collaboration }\end{array}$ & Exercise \\
\hline Artificial intelligence [33] & Practice & Goal & Feedback & Exercise \\
\hline Robotic [26] & $\begin{array}{c}\text { Simulator } \\
\text { Practice } \\
\text { Multimedia }\end{array}$ & Goal & Feedback & Exercise \\
\hline Network security [49] & Ontology & $\begin{array}{c}\text { Goal } \\
\text { Experience } \\
\text { Preference }\end{array}$ & Feedback & Test \\
\hline System analysis $[8,48]$ & $\begin{array}{c}\text { Game } \\
\text { Multimedia }\end{array}$ & Goal & $\begin{array}{l}\text { Annotation } \\
\text { Feedback }\end{array}$ & Test \\
\hline Information retrieval [22] & $\begin{array}{c}\text { Simulator } \\
\text { Multimedia }\end{array}$ & Goal & Feedback & \\
\hline Rough sets (ORS) & $\begin{array}{c}\text { Multimedia } \\
\text { Estimated time }\end{array}$ & $\begin{array}{c}\text { Goal } \\
\text { Experience } \\
\text { Preference } \\
\text { Performance }\end{array}$ & $\begin{array}{c}\text { Collaboration } \\
\text { Feedback }\end{array}$ & Test \\
\hline
\end{tabular}

the complexity of learning support and the interaction support. Both ORS and the system for network security emphasize the adaptability support. However, the ORS improves this feature by considering student performance and providing queries on multiple concepts. Besides, the ORS offers estimated time for students to schedule their learning paces and improve learning efficiency. 


\section{Chapter 5}

\section{Conclusion}

This chapter overviews this research, summarizes the contributions, and proposes some future research directions.

\subsection{Concluding Remarks}

The Web is capable to provide global accessibility and learner-friendly interfaces. Web-based learning offers several advantages compared to traditional learning environments. In post-secondary education, some Web-based learning systems have been built for computer science subjects. However, there are no such systems for rough sets in the literature.

The goal of this research is to design and prototype a Web-based learning support system, named Online Rough Sets, for rough sets. The WLSS offer Web-based learning environments for teachers and students in education. A student-centered learning approach, andragogy, is suitable to rough sets learners in terms of their age. With a learning model based on that approach, learner goals, experience, preference, and performance are considered by the system in the learning process. 
Online Rough Sets supports students to learn the subject in a student-centered learning environment through the Web anytime and anywhere. Moreover, it addresses some limitations found in existing systems to provide personalized study plans to help students learn rationally and efficiently. In addition, this system can be used to learn other subjects by changing learning materials through its graphical interfaces.

\subsection{Summary of Contributions}

The following is the summary of contributions.

\section{Identified a hierarchical structure for rough sets concepts}

The content of ORS was mainly developed from notes, assignments, and exams of a graduate course (CS836) offered at the University of Regina. Based on these materials, we identified a set of concepts with a hierarchical structure, and collected a set of concept-specific quizzes. These concepts were used as learning topics for rough sets. The hierarchical structure contained learning order relationships among concepts, which define prerequisites for each concept. Performance on a quiz indicated student understanding of its related concept. With such hierarchical structure, the concepts and quizzes were applied to ORS as dependent learning materials which would be appropriate to serve different levels of learners.

\section{Developed a student-centered learning model for rough sets learners} As a student-centered learning approach for adults, andragogy was chosen and reviewed since most of rough sets learners were adults. The eight-step learning process of andragogy was used to develop a learning model for those learners. 
The learning model was designed to generate adapted learning sequences for each learner according to the goal, experience, preference, and performance. These personalized learning sequences were used to guide learners to study concepts from bottom to top with satisfying the learning order relationships among concepts. Components of the model were defined and formulated.

\section{Proposed a mechanism to estimate learning time}

The idea of estimating future learning time for each learner is to schedule learning paces. By offering a time slot for each concept, the estimated time is helpful for a learner to allocate proper time to study the concept. A mechanism with cluster analysis and moving average algorithms was proposed to generate the estimated time. For each new learner, the cluster analysis identified the actual learning time of those previous learners having similar personality with this learner. In the actual time, each concept had a series of data distributed on different dates. For each concept, the moving average calculated the average time from the most recent $\mathrm{N}$ pieces of the concept's actual time. Averages were presented to the new learner as time slots in a study plan.

\section{Built a prototype system with the learning model}

A prototype of ORS was built with the learning model and the mechanism for estimated time. Two Java-based open source systems, appfuse and weka, were used in implementation. Appfuse offered the project skeleton and weka provided the APIs of its clustering algorithms. After implementation, the dependent learning materials were input into the system. Two demonstrative examples were performed to show how the system worked and what study plans were 
generated after adaptation. In these examples, adapted study plans were helpful to improve learner effectiveness and efficiency in learning rough sets.

\subsection{Future Research}

An empirical study on ORS is a direction of future research. We plan to utilize the system in future rough sets graduate classes to further evaluate its effectiveness and efficiency. The students can be divided into two groups. One group utilizes ORS to supplement their study, while the other group only depends on the lectures. The final scores of the two groups can be analyzed to examine the effectiveness of ORS.

Another direction is to improve the accuracy of estimated time. The estimated time is predicted by clustering and moving average algorithms from actual learning time. After being used by many learners, the ORS can store the difference between actual time and estimated time as samples of a training set. These samples may lead to the change of algorithm profile for reducing such difference for new learners.

Mouse movement tracking may be applied to record the actual learning time of each concept. The ORS roughly logs the actual time through a mechanism combing text notification and student confirmation. Enhanced by mouse movement tracking, the system may have better opportunity to know whether or not students are learning the subject. This may result more accurate actual time as well as estimated time.

A mobile interface for ORS can be considered for the sake of usability. The presentation layer is originally designed to fit the size and operation behaviors of browsers on PCs and laptops. It may cause some problems when being accessed through a mobile. Since the number of mobile devices keeps on increasing, some learners would prefer mobile devices when using the system. 
Finally, I hope this research is beneficial to people in the community of rough sets, especially graduate students and junior researchers. 


\section{References}

[1] N. Azam and J.T. Yao. Classifying attributes with game-theoretic rough sets. In J. Watada, T. Watanabe, G. Phillips-Wren, R.J. Howlett, and L.C. Jain, editors, Intelligent Decision Technologies, volume 15 of Smart Innovation, Systems and Technologies, pages 175-184. Springer, Berlin Heidelberg, 2012.

[2] N. Azam and J.T. Yao. Game-theoretic rough sets for feature selection. In A. Skowron and Z. Suraj, editors, Rough Sets and Intelligent Systems - Professor Zdzistaw Pawlak in Memoriam, volume 43 of Intelligent Systems Reference Library, pages 61-78. Springer, Berlin Heidelberg, 2013.

[3] N. Azam and J.T. Yao. Analyzing uncertainties of probabilistic rough set regions with game-theoretic rough sets. International Journal of Approximate Reasoning, 55(1):142-155, 2014.

[4] B.P. Bailey and J.A. Konstan. On the need for attention-aware systems: Measuring effects of interruption on task performance, error rate, and affective state. Computers in Human Behavior, 22(4):685-708, 2006.

[5] L.C. Blondy. Evaluation and application of andragogical assumptions to the adult online learning environment. Journal of interactive online learning, 6(2):116-130, 2007.

[6] B. Bomsdorf. Adaptation of learning spaces: Supporting ubiquitous learning in higher distance education. In Mobile Computing and Ambient Intelligence: The Challenge of Multimedia, volume 05181, pages 1-13, Dagstuhl, Germany, 2005.

[7] C.M. Chen, H.M. Lee, and Y.H. Chen. Personalized e-learning system using item response theory. Computers \& Education, 44(3):237-255, 2005.

[8] C.H. Cheng and C.H. Su. A game-based learning system for improving student's learning effectiveness in system analysis course. Procedia - Social and Behavioral Sciences, 31(0):669-675, 2012.

[9] R.S. Clair. Andragogy revisited: Theory for the 21st century. ERIC Clearinghouse on Adult, Career, and Vocational Education, Center on Education and Training for Employment, College of Education, the Ohio State University, 2002. 
[10] A.T. Corbett and J.R. Anderson. Knowledge decomposition and subgoal reification in the act programming tutor. In Proceedings of Artificial Intelligence and Education 95, Charlottesville, 1995.

[11] X.F. Deng and Y.Y. Yao. An information-theoretic interpretation of thresholds in probabilistic rough sets. In T.R. Li, H. Nguyen, G.Y. Wang, J. GrzymalaBusse, R. Janicki, A. Hassanien, and H. Yu, editors, Rough Sets and Knowledge Technology, volume 7414 of Lecture Notes in Computer Science, pages 369-378. Springer, Berlin Heidelberg, 2012.

[12] P.F. Drucker. Managing in the next society. Routledge, London, 2007.

[13] V. Estivill-Castro. Why so many clustering algorithms: a position paper. $A C M$ SIGKDD Explorations Newsletter, 4(1):65-75, 2002.

[14] L. Fan. Web-based learning support system. In J.T. Yao, editor, Web-based Support Systems, volume XXII of Advanced Information and Knowledge Processing, pages 81-95. Springer, London, 2010.

[15] L. Fan and Y.Y. Yao. Web-based learning support systems. In Proceedings of the WI/IAT 2003 Workshop on Applications, Products and Services of Web-based Support systems, pages 43-48, Halifax, 2003.

[16] S. Fleissner, Y. Chan, T.H. Yuen, and V. Ng. Webquest markup language (wqml) for sharable inquiry-based learning. In M. Gavrilova, O. Gervasi, V. Kumar, C.J.K. Tan, D. Taniar, A. Lagana, Y. Mun, and H. Choo, editors, Computational Science and Its Applications - ICCSA 2006, volume 3980 of Lecture Notes in Computer Science, pages 383-392. Springer, Berlin Heidelberg, 2006.

[17] E.C. Freuder and R.J. Wallace. Partial constraint satisfaction. Artificial Intelligence, 58(13):21-70, 1992.

[18] W.A. Fuller. Introduction to statistical time series, volume 428. John Wiley \& Sons, New Jersey, 2009.

[19] E. Gamma, R. Helm, R. Johnson, and J. Vlissides. Design patterns: elements of reusable object-oriented software, volume 49. Addison-Wesley, Boston, 1994.

[20] E. Georgieva, A. Smrikarov, and T. Georgiev. A general classification of mobile learning systems. In Proceedings of CompSys Tech'2005, volume 8, pages IV14.1IV14.6, 2005.

[21] M. Hamada and S. Sato. A game-based learning system for theory of computation using lego nxt robot. Procedia Computer Science, 4(0):1944-1952, 2011. 
[22] E. Herrera-Viedma, A.G. Lpez-Herrera, S. Alonso, J.M. Moreno, F.J. Cabrerizo, and C. Porcel. A computer-supported learning system to help teachers to teach fuzzy information retrieval systems. Information Retrieval, 12(2):179-200, 2009.

[23] R. Hiemstra and B. Sisco. Individualizing instruction. Jossey-Bass, San Francisco, 1990.

[24] C.C. Holt. Forecasting seasonals and trends by exponentially weighted moving averages. International Journal of Forecasting, 20(1):5-10, 2004.

[25] E.F. Holton, R.A. Swanson, and S.S. Naquin. Andragogy in practice: Clarifying the andragogical model of adult learning. Performance Improvement Quarterly, 14(1):118-143, 2001.

[26] S.H. Hong, J.H. Park, K.H. Kwon, and J.W. Jeon. A distance learning system for robotics. In Y. Shi, G. van Albada, J. Dongarra, and P.A. Sloot, editors, Computational Science - ICCS 200\%, volume 4489 of Lecture Notes in Computer Science, pages 523-530. Springer, Berlin Heidelberg, 2007.

[27] H.M. Huang. Toward constructivism for adult learners in online learning environments. British Journal of Educational Technology, 33(1):27-37, 2002.

[28] H. Imaizumi, T. Nagata, G. Kunito, K. Yamazaki, and H. Morikawa. Power saving technique based on simple moving average for multi-channel ethernet. In Proc. 14th OptoElectronics and Communications Conference (OECC 2009), volume 47, pages 1-2, Hong Kong, 2009.

[29] P.A. Jackson. Integrating information literacy into blackboard: Building campus partnerships for successful student learning. The Journal of Academic Librarianship, 33(4):454-461, 2007.

[30] D.W. Kim and J.T. Yao. A web-based learning support system for inquirybased learning. In J.T. Yao, editor, Web-based Support Systems, volume XXII of Advanced Information and Knowledge Processing, pages 125-143. Springer, London, 2010.

[31] R. Kirkby, E. Frank, and P. Reutemann. WEKA Explorer User Guide for Version 3-5-8. University of Waikato, Hamilton, 2008.

[32] M.S. Knowles. The modern practice of adult education, volume 41. New York Association Press, New York, 1970.

[33] M.M. Kozak. Teaching artificial intelligence using web-based applications. J. Comput. Sci. Coll., 22(1):46-53, 2006. 
[34] S. Kumar and M.T.U. Haider. Designing ontology-based academic course for theory of computationto facilitate personalized e-learning system. International Journal of Application or Innovation in Engineering and Management (IJAIEM), 2(3):374-378, 2013.

[35] P. Lingras, P. Bhalchandra, C. Butz, and S. Asharaf. Rough support vectors: Classification, regression, clustering. In A. Skowron and Z. Suraj, editors, Rough Sets and Intelligent Systems - Professor Zdzistaw Pawlak in Memoriam, volume 42 of Intelligent Systems Reference Library, pages 491-515. Springer, Berlin Heidelberg, 2013.

[36] J. McKimm, C. Jollie, and P. Cantillon. Abc of learning and teaching: Web based learning. BMJ: British medical journal, 326(7394):870-873, 2003.

[37] S.B. Merriam. Andragogy and self-directed learning: Pillars of adult learning theory. New Directions for Adult and Continuing Education, 2001(89):3-14, 2001.

[38] J.L. Moore, D.D. Camille, and K. Galyen. e-learning, online learning, and distance learning environments: Are they the same? The Internet and Higher Education, 14(2):129-135, 2011.

[39] M.A.O. Mukhtar, F.B. Mohd Hassan, J.B. Jaafar, and L.A. Rahim. Enhanced approach for developing web applications using model driven architecture. In Research and Innovation in Information Systems (ICRIIS), 2013 International Conference on, pages 145-150, Kuala Lumpur, 2013.

[40] K. Overby. Student-centered learning. ESSAI, 9(1):109-112, 2011.

[41] C.A. Papandreou and D.X. Adamopoulos. Modelling a multimedia communication system for education and training. Computer Communications, 21(6):584589, 1998.

[42] Z. Pawlak and A. Skowron. Rough membership functions. In R.R. Yager, J. Kacprzyk, and M. Fedrizzi, editors, Advances in the Dempster-Shafer theory of evidence, pages 251-271. John Wiley \& Sons, New York, 1994.

[43] O.O. Philip. First, there was pedagogy and then came andragogy. Einstein J. Biol. Med, 21(2):83-87, 2005.

[44] C. Radtke and S. Steiner. Is online learning effective with 9-12 students? In 21st Annual Conference on Distance Teaching and Learning. Retrieved July, volume 16, pages 1-5, Madison, 2005.

[45] M.J. Rosenberg. E-learning: Strategies for delivering knowledge in the digital age, volume 3. McGraw-Hill, New York, 2001. 
[46] J.G. Ruiz, M.J. Mintzer, and R.M. Leipzig. The impact of e-learning in medical education. Academic medicine, 81(3):207-212, 2006.

[47] S. Russell and P. Norvig. Artificial Intelligence: A Modern Approach. Prentice Hall, New Jersey, 2009.

[48] C.H. Su and C.H. Cheng. A game-based learning system for improving student's learning effectiveness in system analysis course. Procedia - Social and Behavioral Sciences, 31(0):669-675, 2012.

[49] Y. Takahashi and T. Abiko. An ontology-based e-learning system for network security. In Advanced Information Networking and Applications, 2005. 19th International Conference on, volume 1, pages 197-202, 2005.

[50] A. Tarhini, K. Hone, and X. Liu. User acceptance towards web-based learning systems: Investigating the role of social, organizational and individual factors in european higher education. Procedia Computer Science, 17(0):189-197, 2013.

[51] J. Turner and K. Bedell. Struts: Kick Start. Sams publishing, Indiana, 2003.

[52] J.J. Vogel, D.S. Vogel, J. Cannon-Bowers, C.A. Bowers, K. Muse, and M. Wright. Computer gaming and interactive simulations for learning: A meta-analysis. Journal of Educational Computing Research, 34(3):229-243, 2006.

[53] K. Wagstaff, C. Cardie, S. Rogers, and S. Schrodl. Constrained k-means clustering with background knowledge. In Proceedings of the Eighteenth International Conference on Machine Learning, volume 1, pages 577-584, San Francisco, 2001.

[54] E. Webb, A. Jones, P. Barker, and P. Schaik. Using e-learning dialogues in higher education. Innovations in Education and Teaching International, 41(1):93-103, 2004.

[55] D. Whitehurst. The AppFuse Primer. Sourcebooks Incorporated, 2008.

[56] R. Xu and D. Wunsch. Survey of clustering algorithms. Neural Networks, IEEE Transactions on, 16(3):645-678, 2005.

[57] J.T. Yao. Web-Based Support Systems, volume XXII. Springer, London, 2010.

[58] J.T. Yao and N. Azam. Incorporating game-theoretic rough sets in webbased medical decision support systems. In Machine Learning and Applications (ICMLA), 2013 12th International Conference on, volume 2, pages 335-338, Miami, 2013.

[59] J.T. Yao and J.P. Herbert. Web-based support systems with rough set analysis. In M. Kryszkiewicz, J.F. Peters, H. Rybinski, and A. Skowron, editors, Rough Sets and Intelligent Systems Paradigms, volume 4585 of Lecture Notes in Computer Science, pages 360-370. Springer, Berlin Heidelberg, 2007. 
[60] J.T. Yao and J.P. Herbert. A game-theoretic perspective on rough set analysis. Journal of Chongqing University of Posts and Telecommunications, 20(3):291298, 2008.

[61] J.T. Yao, D.W. Kim, and J.P. Herbert. Supporting online learning with games. In Proc. SPIE 6570, Data Mining, Intrusion Detection, Information Assurance, and Data Networks Security, volume 6570, pages 65700G-65700G-11, Orlando, 2007.

[62] J.T. Yao and Y.Y. Yao. Web-based support systems. In Proceedings of 2003 WI/IAT Workshop on Applications, Products and Services of Web-based Support System, pages 1-5, Halifax, 2003.

[63] J.T. Yao and Y. Zhang. A scientometrics study of rough sets in three decades. In P. Lingras, M. Wolski, C. Cornelis, S. Mitra, and P. Wasilewski, editors, RSKT 2013, volume 8171 of Lecture Notes in Computer Science, pages 28-40. Springer, Berlin Heidelberg, 2013.

[64] Y.Y. Yao. Three perspectives of granular computing. Journal of Nanchang Institute of Technology, 25(2):16-21, 2006.

[65] Y.Y. Yao. Decision-theoretic rough set models. In J.T. Yao, P. Lingras, W.Z. Wu, M. Szczuka, N.J. Cercone, and D. Slezak, editors, Rough Sets and Knowledge Technology, volume 4481 of Lecture Notes in Computer Science, pages 1-12. Springer, Berlin Heidelberg, 2007.

[66] Y.Y. Yao. Probabilistic rough set approximations. International Journal of Approximate Reasoning, 49(2):255-271, 2008.

[67] Y.Y. Yao. Three-way decisions with probabilistic rough sets. Information Sciences, 180(3):341-353, 2010.

[68] Y.Y. Yao. The superiority of three-way decisions in probabilistic rough set models. Information Sciences, 181(6):1080-1096, 2011.

[69] Y.Y. Yao. Two semantic issues in a probabilistic rough set model. Fundamenta Informaticae, 108(3):249-265, 2011.

[70] Y.Y. Yao and S.K.M. Wong. A decision theoretic framework for approximating concepts. International Journal of Man-Machine Studies, 37(6):793-809, 1992.

[71] F.Y. Yu, Y.H. Liu, and T.W. Chan. A web-based learning system for questionposing and peer assessment. Innovations in Education and Teaching International, 42(4):337-348, 2005.

[72] P. Zdzislaw. Rough sets. International Journal of Computer 85 Information Sciences, 11(5):341-356, 1982. 